\title{
Titanium-Water Loop Heat Pipe Operating Characteristics Under Standard and Elevated Acceleration Fields
}

\author{
Andrew J. Fleming ${ }^{1}$ \\ U.S. Air Force Research Laboratory, Wright-Patterson Air Force Base, Ohio 45433 \\ Scott K. Thomas ${ }^{2}$ \\ Wright State University, Dayton, Ohio 45435 \\ Kirk L. Yerkes ${ }^{3}$ \\ U.S. Air Force Research Laboratory, Wright-Patterson Air Force Base, Ohio 45433
}

An experiment has been developed to examine the behavior of a titanium-water loop heat pipe (LHP) under standard and elevated acceleration fields. The LHP was mounted on a $2.44 \mathrm{~m}$ diameter centrifuge table on edge with heat applied to the evaporator via a mica heater and heat rejected using a high-temperature polyalphaolefin oil (PAO) coolant loop. The LHP was tested under the following parametric ranges: heat load at the evaporator, 100 $\leq Q_{\text {in }} \leq 600 \mathrm{~W}$; heat load at the compensation chamber, $0 \leq Q_{\mathrm{cc}} \leq 50 \mathrm{~W}$; radial acceleration, 0 $\leq a_{\mathrm{r}} \leq 10 \mathrm{~g}$. For stationary operation, the evaporative heat transfer coefficient decreased monotonically with heat load while the thermal resistance decreased to a minimum then increased. Heat input to the compensation chamber was found to increase the evaporative heat transfer coefficient and decrease the thermal resistance for $Q_{\text {in }}=500 \mathrm{~W}$. Transient periodic flow reversal in the LHP was found for some cases, which was likely due to vapor bubble formation in the primary wick. Operation in an elevated acceleration environment revealed that dry-out was dependent on both $Q_{\text {in }}$ and $a_{\mathrm{r}}$, and the ability for the LHP to reprime after an acceleration event that induced dry-out was influenced by the evaporator temperature. The evaporative heat transfer coefficient and thermal resistance were found not to be significantly dependent on radial acceleration. However, the evaporator wall superheat was found to increase slightly with radial acceleration at high heat loads.

\footnotetext{
${ }^{1}$ Mechanical Engineer, 1950 Fifth Street, Building 18.

${ }^{2}$ Associate Professor, Department of Mechanical and Materials Engineering, Associate Fellow AIAA.

${ }^{3}$ Research Engineer, Deputy for Science, 1950 Fifth Street, Building 18. Senior Member AIAA.
} 


\section{Nomenclature}

$$
\begin{aligned}
a & =\text { acceleration, } \mathrm{m} / \mathrm{s}^{2} \\
C_{\mathrm{p}} & =\text { Specific heat, } \mathrm{J} /(\mathrm{kg}-\mathrm{K}) \\
D & =\text { Diameter, } \mathrm{m} \\
f & =\text { Frequency, } \mathrm{Hz} \\
g & =\text { Standard acceleration, } 9.81 \mathrm{~m} / \mathrm{s}^{2} \\
h & =\text { Heat transfer coefficient, } \mathrm{W} /\left(\mathrm{m}^{2}-\mathrm{K}\right) \\
k & =\text { Thermal conductivity, } \mathrm{W} /(\mathrm{m}-\mathrm{K}) \\
L & =\text { Length, } \mathrm{m} \\
m & =\text { Mass, } \mathrm{kg} \\
\mathrm{Nu} & =\text { Nusselt number, } h D / k \\
Q & =\text { Heat transfer rate, } \mathrm{W} \\
r & =\text { Radial coordinate, } \mathrm{m} \\
R & =\text { Thermal resistance, } \mathrm{K} / \mathrm{W} \\
\mathrm{Ra} & =\text { Rayleigh number, } g \beta\left(T_{s}-T_{\infty}\right) D^{3} / v \alpha \\
t & =\text { Time, } \mathrm{s} \\
T & =\text { Temperature, } \mathrm{K} \\
V & \text { Average temperature, } \mathrm{K} \\
T & =\text { Volume, } \mathrm{m}^{3} \\
& =1
\end{aligned}
$$

Greek Letters

$$
\begin{aligned}
& \alpha=\text { Thermal diffusivity, } \mathrm{m}^{2} / \mathrm{s} \\
& \beta=\text { Volumetric thermal expansion coefficient, } \mathrm{K}^{-1} \\
& \gamma=\text { Ratio of specific heats } \\
& \Delta T=\text { Temperature difference, } \mathrm{K} \\
& \Delta T_{\mathrm{sh}}=\text { Evaporator wall superheat, } \bar{T}_{\mathrm{e}}-T_{\mathrm{e} / \mathrm{cc}}, \mathrm{K} \\
& \varepsilon \quad=\quad \text { Emissivity } \\
& \theta \quad=\text { Resultant acceleration vector angle, arc degrees } \\
& \mu \quad=\text { Absolute viscosity, }(\mathrm{N}-\mathrm{s}) / \mathrm{m}^{2}
\end{aligned}
$$




$$
\begin{aligned}
v & =\text { Kinematic viscosity, } \mathrm{m}^{2} / \mathrm{s} \\
\rho & =\text { Density, } \mathrm{kg} / \mathrm{m}^{3} \\
\sigma & =\text { Stefan-Boltzmann constant, } 5.67 \times 10^{-8} \mathrm{~W} /\left(\mathrm{m}^{2}-\mathrm{K}^{4}\right) \\
\varphi & =\text { Fluid phase }
\end{aligned}
$$

\section{Subscripts}

$1=$ Primary

$2=$ Secondary

$\mathrm{b}=$ Bayonet inlet

$\mathrm{c} \quad=$ Condenser

$\mathrm{cc}=$ Compensation chamber

$\mathrm{cl}=$ Centerline

conv $=$ Convection

$\mathrm{cp}=$ Cold plate

$\mathrm{D}=$ Diameter

$\mathrm{e} \quad=\quad$ Evaporator

eg $=$ Ethylene glycol

$\mathrm{e} / \mathrm{cc}=$ Evaporator/compensation chamber junction

ie $\quad=\quad$ Loop heat pipe inner edge

in $\quad=\quad$ In

$\max =$ Maximum

oe $\quad=\quad$ Loop heat pipe outer edge

out $=$ Out

PAO $=$ Polyalphaolefin

$\mathrm{r} \quad=\quad$ Radial

$\operatorname{rad}=$ Radiation

sh = Superheat

tot $=$ Total

$\mathrm{v}=$ Vapor 
$\mathrm{z} \quad=\quad$ Axial

\section{Introduction}

OOP heat pipes (LHP's) are two-phase thermal transport devices that operate passively using the latent heat of vaporization to transport heat from one location to another. The LHP was invented in 1972 by Gerasimov and Maidanik [1] in the former Soviet Union, and was later patented in the United States [2]. The LHP consists of an evaporator, compensation chamber, liquid and vapor transport lines made of smooth tubing, and a condenser as shown in Fig. 1a. Heat is applied directly to the exterior wall of the evaporator, which often has a circular crosssection. The majority of the input heat is used to vaporize the working fluid within the primary wick structure, which is an inverted meniscus wick in direct contact with the exterior evaporator wall. The vapor is captured in the axial vapor grooves in the primary wick and is directed via a manifold at the end of the evaporator to the vapor line due to the increased pressure within the evaporator. Menisci are developed in the primary wick due to evaporation which establishes a capillary pressure head that returns liquid to the evaporator from the condenser. This capillary head must be greater than the total system pressure drop in order for the LHP to continue to operate without drying out.

The vapor from the evaporator section travels via the vapor line to the condenser section, which is also made of smooth tubing. Heat is rejected from the condenser to the ultimate heat sink. The working fluid enters the condenser as a superheated vapor. After sufficient heat is rejected, the vapor becomes a saturated vapor, a two-phase mixture, a saturated liquid, and, depending on the amount of heat rejection, it may or may not become a subcooled liquid. The location of the point at which the working fluid becomes a subcooled liquid $(2 \varphi-1 \varphi$ point $)$ is dependent on the heat input at the evaporator, the heat rejection at the condenser, and the saturation temperature in the compensation chamber. After exiting the condenser section, the liquid will continue to lose heat due to convection and/or thermal radiation to the ambient. The subcooled liquid returns to the evaporator via the bayonet tube, which delivers the liquid to the end of the evaporator where the vapor manifold resides.

As stated previously, most of the evaporator heat input evaporates liquid in the primary wick. The rest of the heat is transferred by conduction through the primary wick, where liquid is evaporated into vapor channels leading to the compensation chamber (Fig. 1b and c). Part of this vapor stream condenses onto the secondary wick, which is in intimate contact with the bayonet tube. This heat transfer to the bayonet tube raises the temperature of the subcooled liquid entering the compensation chamber to the saturation temperature as it travels to the end of the evaporator. The 
rest of the vapor condenses onto the wick lining the compensation chamber. This latent heat is then rejected from the compensation chamber to the ambient. The condensate in the compensation chamber is drawn back to the evaporator section through the secondary wick by capillary action. In this way, the secondary wick and the compensation chamber behave similar to a conventional heat pipe.

The compensation chamber allows the LHP to automatically regulate itself during startup, shutdown, or a change in the operating conditions. The compensation chamber provides for storage of excess liquid when the evaporator heat input is high, where the majority of the condenser section is free of subcooled liquid. The compensation chamber can also be used to control the location of the $2 \varphi-1 \varphi$ point in the condenser. Controlling the heat transfer through the shell of the compensation chamber can adjust the saturation point in the condenser, thereby changing the amount of subcooling of the liquid returning to the evaporator.

There has been limited experimentation on the acceleration effects on loop heat pipes and heat pipes. Ku et al. [4] performed experiments on a miniature aluminum/anhydrous ammonia LHP by using a spin table to examine the effects of varying acceleration on start-up. Four mounting configurations were examined: horizontally with the compensation chamber and liquid line outboard on the table, horizontally with the evaporator and vapor line outboard on the table, vertically with evaporator above the compensation chamber with no radial acceleration, and vertically with evaporator below the compensation chamber with no radial acceleration. Several different experiments were conducted, including LHP startup before acceleration was applied and vice versa, as well as varying heat load inputs up to $Q_{\mathrm{in}}=100 \mathrm{~W}$. Several acceleration profiles were examined, including $a_{\mathrm{r}}=0.0 \mathrm{~g}$, constant $a_{\mathrm{r}}=1.2 \mathrm{~g}$, constant $a_{\mathrm{r}}=4.8 \mathrm{~g}$, combination of constant $a_{\mathrm{r}}=1.2$ and $4.8 \mathrm{~g}$, constant $a_{\mathrm{r}}=1.2 \mathrm{~g}$ for 30 seconds followed by $a_{\mathrm{r}}=0.0 \mathrm{~g}$ for 300 seconds periodically, constant $a_{\mathrm{r}}=4.8 \mathrm{~g}$ for 30 seconds followed by $a_{\mathrm{r}}=0.0 \mathrm{~g}$ for 300 seconds periodically, and combinations of $a_{\mathrm{r}}=1.2$ and $4.8 \mathrm{~g}$ followed by $a_{\mathrm{r}}=0.0 \mathrm{~g}$ for 300 seconds periodically. Their experimental results indicated that the wall superheat, defined as the difference between the evaporator and compensation chamber wall temperatures, appeared to be independent of input heat load and acceleration. When temperature overshoot in the evaporator was examined, for heat loads greater than $Q_{\mathrm{in}}=50 \mathrm{~W}$, there was essentially no overshoot. For smaller heat loads, such as at $Q_{\text {in }}=5 \mathrm{~W}$, a temperature overshoot of a few degrees was always observed, but at $Q_{\text {in }}=25 \mathrm{~W}$, the temperature overshoot ranged from 0 to $45^{\circ} \mathrm{C}$. In every experiment, the LHP started successfully. 
$\mathrm{Ku}$ et al. [5], in an extension of the previous experimental study, examined the temperature stability of the same miniature LHP under varying heat loads and acceleration levels. Their experimental results showed that the radial acceleration caused a redistribution of fluid in the evaporator, condenser, and compensation chamber, which in turn changed the LHP operating temperature. The effect was not universal, in the sense that all of the operating conditions needed to be taken into account. With sufficient time, constant acceleration could either increase or decrease the LHP operating temperature. Periodic acceleration led to a quasi-steady operating temperature. Temperature hysteresis could also be caused by the radial acceleration. In all of the experiments the LHP continued to operate without problems.

Similar research has been conducted to examine body force effects on heat pipes. Ponnappan et al. [6] examined a flexible copper-water arterial wick heat pipe subjected to transverse acceleration using a centrifuge table. Evaporator heat loads up to $Q_{\mathrm{in}}=150 \mathrm{~W}$ and steady state radial accelerations up to $a_{\mathrm{r}}=10.0 \mathrm{~g}$ were investigated. Transport capacity of the heat pipe dropped from $Q_{\text {out }}=138 \mathrm{~W}$ at radial accelerations of $a_{\mathrm{r}}=1.0 \mathrm{~g}$ to $Q_{\text {out }}=60 \mathrm{~W}$ at $a_{\mathrm{r}}=10.0 \mathrm{~g}$. The temperature difference between the evaporator and condenser remained fairly constant up to $a_{\mathrm{r}}=4.0$ $\mathrm{g}$ then decreased from $a_{\mathrm{r}}=4.0$ to $10.0 \mathrm{~g}$. This decrease was due to a more uniform distribution of fluid within the wick at the higher radial acceleration.

Yerkes and Beam [7] examined the same flexible copper-water arterial wick heat pipe as Ponnappan et al. under transient transverse and axial acceleration forces with periodic and burst transverse accelerations from $f=0.01$ to $0.03 \mathrm{~Hz}$ and magnitudes from $a_{\mathrm{r}}=1.1$ to $9.8 \mathrm{~g}$ peak-to-peak and evaporator heat inputs up to $Q_{\text {in }}=83 \mathrm{~W}$. It was observed that pooling of excess fluid had a significant effect on the heat transport of the heat pipe at steady state transverse acceleration. Heat transport potential decreased with increasing transverse acceleration, which was caused by partial dry-out of the artery and pooling in the condenser. The heat pipe was able to reprime after dry-out events with subsequent reduction of transverse acceleration. Under cyclic transverse acceleration, significant fluid slosh was thought to create a cyclic variation in heat pipe temperature. Temperature rise was lower at the onset of dry-out conditions when compared to steady state transverse acceleration. Frequency of the steady periodic burst transverse acceleration had no effect on the heat pipe temperature and tended to delay the onset of dry-out.

Thomas and Yerkes [8] examined the same flexible copper-water arterial wick heat pipe as Ponnappan et al. with evaporator heat loads from $Q_{\mathrm{in}}=75$ to $150 \mathrm{~W}$, condenser temperatures of $T_{\mathrm{c}}=3,20$, and $35^{\circ} \mathrm{C}$, and sinusoidal acceleration frequencies of $f=0,0.01,0.05,0.1,0.15$, and $0.2 \mathrm{~Hz}$. The amplitude of the radial acceleration ranged 
from $a_{\mathrm{r}}=1.1$ to $9.8 \mathrm{~g}$. The effects of the previous dry-out history of the heat pipe were also examined. It was discovered that the thermal resistance increased and then decreased with respect to increasing acceleration frequency. The thermal resistance also increased with increasing evaporator heat loads. The previous dry-out history adversely affected the thermal resistance of the heat pipe when dry-out occurred prior to increasing the acceleration frequency.

Thomas et al. [9] examined the behavior of a helically grooved copper-ethanol heat pipe in terms of the evaporator heat input and transverse radial acceleration. Heat loads ranging from $Q_{\text {in }}=20$ to $250 \mathrm{~W}$ were applied to the evaporator. At $Q_{\mathrm{in}}=20 \mathrm{~W}$ the heat pipe did not experience any dry-out conditions when the radial acceleration was increased and then decreased stepwise from $a_{\mathrm{r}}=0$ to $10 \mathrm{~g}$. At $Q_{\text {in }}=50 \mathrm{~W}$, the heat pipe experienced dry-out conditions at $a_{\mathrm{r}}=0.0$ and $2.0 \mathrm{~g}$, but quickly reprimed at the higher radial accelerations. This indicated the elevated body forces actually aided the performance of the heat pipe by increasing the capillary limit due to the forces generated from acceleration gradients down the length of the helical groove. The thermal resistance of the heat pipe was noted to decrease then increase with increasing heat transported when dry-out started.

Zaghdoudi and Sarno [10] examined the body force effects on a flat copper-water heat pipe via a centrifuge setup. The heat pipe was mounted such that the accelerating forces were opposite to the liquid flow, or in an "unfavorable" mounting condition. Three types of acceleration profiles were performed in this study: A parabolic profile from $a_{\mathrm{r}}=0.0$ to 10.0 to $0.0 \mathrm{~g}$ with a 5 second stabilization at $a_{\mathrm{r}}=10.0 \mathrm{~g}$, a step increase from $a_{\mathrm{r}}=0.0$ to 10.0 to $0.0 \mathrm{~g}$ with a 10 second stabilization at each step, and increasing then decreasing the acceleration from $a_{\mathrm{r}}=0.0$ to $10.0 \mathrm{~g}$ after thermal stabilization. Heat loads of $Q_{\text {in }}=20,40$, and $60 \mathrm{~W}$ were applied to examine the effect on evaporator and condenser temperatures as well as to determine the thermal resistance. For the first two types of acceleration profile, a delayed increase in evaporator temperature and a decrease in condenser temperature was observed. This was likely due to pooling of fluid in the condenser. Thermal resistance also experienced a delayed onset and remained elevated even in the absence of an accelerating force. For the third type of acceleration profile, there was a much more gradual increase in evaporator temperature and nearly negligible decrease in condenser temperature, quickly returning to normal in the absence of the accelerating force. Thermal resistance had a similar trend, quickly returning to normal after the acceleration burst. This suggested that the heat pipe quickly reprimed after the acceleration event. These tests demonstrated the importance of prior operation history when the heat pipe was subjected to elevated body forces. 
The objective of the present experiment was to determine the operating characteristics of a titanium-water loop heat pipe subjected to varying heat loads and radial acceleration levels. The titanium-water LHP configuration was selected for high-temperature electronics applications as given by Anderson et al. [11]. Transient temperature distributions, the evaporative heat transfer coefficient, the thermal resistance, and the evaporator wall superheat have been found in terms of the heat input at the evaporator, heat input at the compensation chamber, and radial acceleration field. In addition, transient behavior during startup and nominally steady operation has been examined. A performance map has been developed that relates dry-out to the heat load and radial acceleration for the experimental conditions described. The experimental parametric ranges were as follows: heat load at the evaporator, $100 \leq Q_{\mathrm{in}} \leq 600 \mathrm{~W}$; heat load at the compensation chamber, $0 \leq Q_{\mathrm{cc}} \leq 50 \mathrm{~W}$; radial acceleration, $0 \leq a_{\mathrm{r}} \leq 10 \mathrm{~g}$.

\section{Experimental Setup}

The Centrifuge Table Test Bed at Wright-Patterson Air Force Base (AFRL/RZPS) was used to determine the heat transfer characteristics of the titanium-water LHP under stationary and elevated acceleration fields. The test bed consisted of a $2.44 \mathrm{~m}$ diameter horizontal rotating table driven by a $20 \mathrm{hp} \mathrm{DC}$ electric motor. The test bed was able to deliver the following to devices mounted to the rotating table: Conditioned DC electrical power through three separate power supplies, $120 \mathrm{VAC}$ power, temperature-controlled ethylene glycol coolant, and electrical signals for analog or digital control. In addition, electrical signals were collected from instruments on the table and stored in a data acquisition computer. The radial acceleration could exceed $a_{\mathrm{r}}=12 \mathrm{~g}$, with a maximum onset of approximately $\dot{a}_{\mathrm{r}}=10 \mathrm{~g} / \mathrm{s}$, inducing a tangential acceleration. The acceleration field could be varied manually using a potentiometer, or controlled digitally using a signal generator in the data acquisition system. The acceleration field was measured using an orthogonal triaxial accelerometer (Columbia SA-307HPTX).

Power was supplied to heaters on the table by three precision power supplies through power slip rings. These slip rings were separated from the instrumentation slip rings to reduce electrical noise. The heater power was calculated by multiplying the voltage drop across the heater by the current. The current was determined from the voltage drop across a precision resistor in series with the heater. This type of measurement was required due to the voltage drop between the control room and the table.

Heat was rejected from the centrifuge table using an ethylene-glycol/water mixture that was delivered to the rotating centrifuge table via a double-pass hydraulic rotary coupling. The temperature of the coolant was maintained 
at a constant setting by a recirculating constant-temperature bath. The volumetric flow rate of the coolant mixture was controlled using a high-pressure booster pump, which aided the low-pressure pump in the recirculating chiller. Throughout experimentation the flow rate was held constant.

Instrumentation signals generated on the table were acquired through a custom-built forty-channel instrumentation slip ring using a data acquisition system. Temperatures, mass flow rates, accelerations, and voltages were all measured using a data acquisition mainframe (Agilent VXI E8408A) with a command module (Agilent E1406A), a 51/2 digit multimeter module (Agilent E1411B), and a 64-channel 3-wire multiplexer module (Agilent E1476A). The rotational speed of the centrifuge table, heater power, and other low voltage control devices on the table were controlled using an 8/16-channel D/A converter module (Agilent E1418A). Communication between the data acquisition unit and the computer was established using a general purpose interface bus coupled with a customdesigned LabVIEW virtual instrument.

Gathering temperature data from rotating machinery using slip rings presents unique problems. First, when thermocouple wires are connected to the wires leading to a slip ring, at least one extra junction is created, depending on the materials of the thermocouple wires. To avoid this problem, a Type E thermocouple amplifier was installed on the centrifuge table with internal cold junction compensation. This converted the millivoltage signals from the thermocouples to 0 to $10 \mathrm{~V}$ signals without the creation of extra junctions. Another problem that is present when slip rings are used is electrical noise. This problem was reduced (not eliminated) by the use of a low-pass filter for each of the thermocouple signals coming from the table before the data acquisition system.

The test article, a titanium-water loop heat pipe, was developed for AFRL/RZPS by Advanced Cooling Technologies, Inc., under contract FA8601-06-P-0076. Initial design parameters set by AFRL/RZPS were to develop a loop heat pipe capable of a minimum heat load of $500 \mathrm{~W}$ and minimum heat flux of $3 \mathrm{~W} / \mathrm{cm}^{2}$. The minimum transport line length was $2 \mathrm{~m}$ to simulate relevant aircraft geometries. An evaporator operating temperature of $200^{\circ} \mathrm{C}$ and condenser operating temperature between 5 and $140^{\circ} \mathrm{C}$ were selected to match relevant acquisition and rejection temperatures aboard aircraft. The LHP was passivated and purged of initially formed noncondensable gas identified during life tests of similar titanium-water heat pipes by Anderson et al. [12]. A summary of the loop heat pipe specifications can be seen in Table 1. The LHP was instrumented with twelve type E exposed tip thermocouples as seen in Fig. 2. A summary of the locations of all of the thermocouples is given in Table 2 . 
The loop heat pipe was mounted onto the centrifuge table such that the centerline of the tubing coincided with the outer table radius as much as possible. Small deviations existed since the condenser section and the evaporator/compensation chamber were both straight. This induced a non-uniform radial acceleration field over the lengths of these sections that needed to be quantified. A survey was taken at 22 locations on the loop heat pipe to determine how far various portions of the loop heat pipe were from the centerline radius. The loop heat pipe had a minimum radius to centerline of $119.2 \mathrm{~cm}$ and a maximum radius to centerline of $123.3 \mathrm{~cm}$. The entire loop heat pipe fitted within $4.6 \mathrm{~cm}$ for a percent acceleration difference of 3.8\% [13]. Stands were designed using G-7 phenolic to mount the loop heat pipe with support at the compensation chamber, evaporator, condenser, and transport lines. The tops of these stands were anchored to the table to reduce deflection when the table was rotating. To minimize heat loss to the environment, the entire assembly was thoroughly insulated using Kaowool blankets and aluminum foil. The assembly was placed inside an aluminum frame for structural support and enclosed with sheet metal sides to minimize convective heat losses.

During operation, heat was applied to the LHP at the evaporator while the heat transfer to the compensation chamber was independently controlled. A mica heater was located between the aluminum evaporator body and a ceramic fiber insulative layer, followed by the evaporator stand. A flexible electric heater tape was wound around the compensation chamber and surrounded by insulation and aluminum foil to minimize heat losses.

As previously mentioned, the centrifuge table was equipped with an on-board fluid loop for dissipating heat from sources on the table, which used ethylene glycol as its working fluid. In the present experiment, it was desired to have the option of operating the LHP condenser section at elevated temperatures, so a high-temperature fluid loop was constructed and mounted to the centrifuge table to act as an interface between the LHP and the low-temperature fluid loop. The high-temperature working fluid (Brayco Micronic 889 polyalphaolefin or PAO oil) flowed from a custom-made copper reservoir into a positive displacement gear pump. After passing through a filter and a flowstraightening section, the PAO was directed through the turbine flow meter (Omega FTB-9506). The calorimeter on the condenser section consisted of three heat exchangers plumbed in series and mounted to the condenser section. Type E thermocouple probes were installed at the inlet and outlet of the three heat exchangers for calorimetry and another was placed prior to the flow meter. This was needed due to the dependence of the viscosity of PAO on temperature. After the PAO exited the three heat exchangers on the condenser, it flowed to a liquid/liquid heat 
exchanger that transferred heat from the high-temperature coolant loop to the low-temperature ethylene glycol loop. The PAO then returned to the reservoir. A schematic of the experimental setup can be seen in Fig. 3.

Thermocouple calibrations were conducted over two temperature ranges depending on the anticipated operating temperatures. Grounded probe thermocouples were used for calorimetry, coolant flow meter calibration and the measurement of the ambient temperature. These four thermocouples were calibrated over the anticipated range of 20 to $145^{\circ} \mathrm{C}$ in $5^{\circ} \mathrm{C}$ intervals. Twelve exposed tip thermocouples were mounted on the LHP in various locations and needed to be calibrated over the full range of 20 to $230^{\circ} \mathrm{C}$ in $5^{\circ} \mathrm{C}$ increments. The calibration procedure consisted of using two separate recirculating constant-temperature baths with PAO as the working fluid to achieve the required temperature range. The temperature readings from the sixteen thermocouples were compared to a NIST-traceable platinum resistance temperature detector (RTD) with a resolution of $\pm 0.009^{\circ} \mathrm{C}$. To ensure that the bath had reached steady state at a given set-point temperature, the RTD temperature was continuously monitored. When the standard deviation of 100 readings dropped below the specified threshold of $0.005^{\circ} \mathrm{C}, 100$ readings from the thermocouples were sampled, stored in an array, and the bath temperature was changed. For repeatability, the bath temperature was first incremented from the lowest temperature to the highest temperature, and then decremented from highest to lowest, and the two sets of 100 data points collected for each thermocouple at a given temperature were used to determine average readings. The uncertainty associated with each thermocouple was determined by accounting for four sources of error: the stated uncertainty of the RTD, the confidence interval of the RTD average reading at a confidence level of 0.95 , the confidence interval of the thermocouple average reading at a confidence level of 0.95 , and the maximum deviation of the temperature calculated using the polynomial curve fit from the actual measured temperature.

The turbine flow meter used in the high-temperature fluid loop was calibrated to achieve accurate results for the amount of heat extracted from the LHP. This was critical for the calculation of the evaporative heat transfer coefficient and the thermal resistance of the LHP. Since the viscosity of the PAO used in the high-temperature fluid loop changes significantly with temperature, a "calibration surface" was generated that related the output voltage of the flow meter and the temperature of the PAO at the entrance of the flow meter to the mass flow rate. The calibration setup consisted of a recirculating constant-temperature bath filled with PAO from the same source as used in the high-temperature fluid loop. The gear pump, inline filter, and calibrated grounded thermocouple probe from the high-temperature fluid loop were installed with tubing from the bath to the turbine flow meter. Flow 
straightening sections upstream and downstream were placed according to the manufacturer's instructions. A threeway valve was installed after the flow meter, which allowed the entire flow system to reach a steady temperature. Once the temperature was steady, the flow was diverted to a catch basin for a specified amount of time. The voltage from the flow meter and the temperature from the thermocouple were recorded during this time, and when the basin was full, the flow was again diverted to recirculating the PAO back to the bath. All of the data was collected through the instrumentation slip rings on the centrifuge table to the data acquisition system to capture all errors inherent to the centrifuge table test bed. A laboratory scale was used to determine the mass collected during a given test run. During each measurement, as many data points as possible were collected from the flow meter across the time span with the limiting factor being the iteration time on the LabVIEW software. The minimum number of data points collected for any given run was 437 . The voltages and temperatures were averaged and a confidence interval was calculated based on a confidence level of 0.95 for each test run. The test was repeated for a total of five averaged data points for each nominal temperature and flow rate. These tests were completed over the range of $T=20$ to $120^{\circ} \mathrm{C}$ in intervals of $25^{\circ} \mathrm{C}$ and flow rates ranging from $\dot{m}_{\mathrm{cp}}=0.0064$ to $0.025 \mathrm{~kg} / \mathrm{s}$ in intervals of approximately $0.002 \mathrm{~kg} / \mathrm{s}$. A 3-D paraboloid regression equation was generated to relate temperature, flow meter voltage, and mass flow rate. The uncertainty of the mass flow rate measurement was affected by the maximum deviation of the regression equation from the actual data, the confidence interval for the temperature and flow meter voltage measurements, the root-sum-square total error associated with the scale and stopwatch and the root-sum-square error associated with the temperature and voltage measurements.

The heat transferred from the LHP condenser to the cold plate was

$$
Q_{\text {out }}=\dot{m}_{\mathrm{cp}} C_{\mathrm{p}, \mathrm{PAO}}\left(T_{\mathrm{out}}-T_{\mathrm{in}}\right)
$$

A linear fit equation for $C_{\mathrm{p}, \mathrm{PAO}}$ as a function of temperature was developed by Ghajar et al. [14] and used in Eq. (1). The average evaporative heat transfer coefficient was defined as

$$
\bar{h} \equiv \frac{Q_{\text {out }}}{\pi D L\left(\bar{T}_{\mathrm{e}}-T_{\mathrm{v}}\right)}
$$

where $D$ is the inside diameter of the evaporator shell, $L$ is the length of the evaporator, $\bar{T}_{\mathrm{e}}$ is the average evaporator temperature measured by the four thermocouples embedded in the wall between the heater and the wick (Fig. 2b), 
and $T_{\mathrm{v}}$ is the external temperature of the vapor line at the outlet of the evaporator. The thermal resistance of the loop heat pipe was determined using the average evaporator temperature and the average temperature of the cold plate

$$
R \equiv \frac{\bar{T}_{\mathrm{e}}-\bar{T}_{\mathrm{cp}}}{Q_{\text {out }}}
$$

where $\bar{T}_{\mathrm{cp}}=0.5\left(T_{\text {out }}+T_{\text {in }}\right)$ is the average cold plate temperature. The root-sum-square uncertainties of $Q_{\text {out }}, \bar{h}$, and $R$ are given by

$$
\begin{gathered}
\Delta Q_{\text {out }}=\left[\left(C_{\mathrm{p}, \mathrm{PAO}}\left(T_{\text {out }}-T_{\mathrm{in}}\right) \Delta \dot{m}_{\mathrm{cp}}\right)^{2}+\left(\dot{m}_{\mathrm{cp}}\left(T_{\text {out }}-T_{\mathrm{in}}\right) \Delta C_{\mathrm{p}, \mathrm{PAO}}\right)^{2}+\left(\dot{m}_{\mathrm{cp}} C_{\mathrm{p}, \mathrm{PAO}} \Delta T_{\mathrm{out}}\right)^{2}\right. \\
\left.+\left(-\dot{m}_{\mathrm{cp}} C_{\mathrm{p}, \mathrm{PAO}} \Delta T_{\mathrm{in}}\right)^{2}\right]^{1 / 2} \\
\Delta \bar{h}=\left[\left(\frac{1}{\pi D L\left(\bar{T}_{\mathrm{e}}-T_{\mathrm{v}}\right)} \Delta Q_{\text {out }}\right)^{2}+\left(\frac{-Q_{\text {out }}}{\pi D^{2} L\left(\bar{T}_{\mathrm{e}}-T_{\mathrm{v}}\right)} \Delta D\right)^{2}+\left(\frac{-Q_{\text {out }}}{\pi D L^{2}\left(\bar{T}_{\mathrm{e}}-T_{\mathrm{v}}\right)} \Delta L\right)^{2}\right. \\
\left.+\left(\frac{-Q_{\text {out }}}{\pi D L\left(\bar{T}_{\mathrm{e}}-T_{\mathrm{v}}\right)^{2}} \Delta \bar{T}_{\mathrm{e}}\right)^{2}+\left(\frac{-Q_{\text {out }}}{\pi D L\left(\bar{T}_{\mathrm{e}}-T_{\mathrm{v}}\right)^{2}} \Delta T_{\mathrm{v}}\right)^{2}\right]^{1 / 2} \\
\Delta R=\left[\left(\frac{-\left(\bar{T}_{\mathrm{e}}-\bar{T}_{\mathrm{cp}}\right)}{Q_{\text {out }}^{2}} \Delta Q_{\text {out }}\right)^{2}+\left(\frac{1}{Q_{\text {out }}} \Delta \bar{T}_{\mathrm{e}}\right)^{2}+\left(\frac{-1}{Q_{\text {out }}} \Delta \bar{T}_{\mathrm{cp}}\right)^{2}\right]^{1 / 2}
\end{gathered}
$$

The uncertainty of $C_{\mathrm{p}, \mathrm{PAO}}$ was estimated by Ghajar et al. to be $0.5 \%$ of the value. For each steady state condition, 151 data points were collected from each sensing device representing five minutes of data. Measured values were averaged and uncertainties were calculated based on the fixed error of each instrument and the confidence interval for the average at a confidence level of 0.95 . A summary of the uncertainties for this experiment can be found in Table 3. Details of the uncertainty analysis are given by Fleming [13].

\section{Results and Discussion}

The purpose of this series of experiments was to determine the operating characteristics of a titanium-water loop heat pipe subjected to changes in evaporator heat input, compensation chamber heat input, and radial acceleration. Steady state and transient temperature data were collected which provided insight into the fluid-thermal behavior of the LHP. The raw data was reduced to obtain the evaporative heat transfer coefficient, thermal resistance, and evaporator wall superheat in terms of the heat transported and radial acceleration level. Quasi-steady phenomena and dry-out of the LHP were observed and quantified in a performance map. 
A typical stationary $\left(a_{\mathrm{z}}=1.0 \mathrm{~g}, a_{\mathrm{r}}=0.0 \mathrm{~g}\right)$ cold-start test of the LHP is presented in Fig. 4, which consisted of the following: with the LHP at ambient conditions, the recirculating constant-temperature bath in the low-temperature fluid loop was set to $T_{\mathrm{eg}}=35^{\circ} \mathrm{C}$. Heat was applied as a step function to the evaporator section (in this case, $Q_{\text {in }}=600$ W) while the pump for the high-temperature fluid loop was simultaneously turned on ( $\dot{m}_{\mathrm{cp}}=0.0077 \mathrm{~kg} / \mathrm{s}$ ). The mass flow rate of the high-temperature fluid loop was maintained constant at this value throughout this series of experiments to minimize the uncertainty associated with the calorimetry of the cold plate. In Fig. 4a, the evaporator temperature (TC04) increased very quickly while the rest of the LHP did not react. After approximately $60 \mathrm{~s}$, the thermocouple located on the vapor line nearest to the exit of the evaporator (TC08) suddenly increased. This was followed in turn by increases in temperature reflected by the thermocouples located throughout the condenser section. This shows the progression of the saturated vapor clearing the condenser section of liquid, which was subsequently displaced into the evaporator section and the compensation chamber via the bayonet tube. Fig. $4 \mathrm{~b}$ shows that the evaporator temperature was significantly higher than the condenser temperatures, which led to a relatively high value of thermal resistance, which will be discussed in detail below. The temperatures appear to become steady after approximately $6000 \mathrm{~s}$. However, in order to determine when steady state occurred the time rate of change of the temperatures was averaged over 15 min. intervals and plotted with respect to time as shown in Fig. 4c. It was observed that $\mathrm{d} T / \mathrm{d} t$ approached zero shortly after $6000 \mathrm{~s}$, but for times greater than $6000 \mathrm{~s}$, significant oscillations occurred. The oscillations in $\mathrm{d} T / \mathrm{d} t$ were not apparent in the raw temperature traces, but steady state was found to occur at approximately $18,000 \mathrm{~s}$. This methodology was used throughout testing to ensure that a repeatable steady state was reached.

Temperature traces in the condenser (TC09 through TC13) and at the bayonet inlet (TC14) are shown in Fig. 5 after the stationary LHP reached steady state conditions at heat inputs ranging from $100 \leq Q_{\text {in }} \leq 600 \mathrm{~W}$. In Fig. 5a, with $Q_{\text {in }}=100 \mathrm{~W}$, the liquid entering the bayonet tube was highly subcooled at approximately $38^{\circ} \mathrm{C}$. At this heat input level, the majority of the condenser was flooded with subcooled liquid. In fact, only TC09 (condenser inlet) indicated two-phase flow. The case when then evaporator heat input was set to $Q_{\text {in }}=200 \mathrm{~W}$, shown in Fig. $5 \mathrm{~b}$, was a unique operating point that is described further in the following paragraph (Fig. 6). Fig. 5c to Fig. 5f shows that the $2 \varphi-1 \varphi$ point progressed through the liquid line as heat input increased until it reached the bayonet inlet. If the heat input at the evaporator is high enough, saturated vapor will pass through the bayonet tube and reach the evaporator 
section. This point represents a performance limit to LHP operation because if vapor enters the evaporator, the wick will dry out and the LHP will overheat.

The oscillatory behavior of the LHP for the heat input of $Q_{\text {in }}=200 \mathrm{~W}$ is shown in Fig. 6. Initially, at $t=0$, the evaporator temperatures (TC04, $\mathrm{TC} 05, \mathrm{TC} 06$, and $\mathrm{TC} 07)$ ranged from 66 to $68^{\circ} \mathrm{C}$. The evaporator temperature nearest to the bayonet tube outlet (TC07) was the lowest, which indicated that the subcooled liquid that entered the evaporator tended to reduce the evaporator temperature at this point. The vapor line and condenser temperatures (TC08 through $\mathrm{TC} 13$ ) ranged from 46 to $58^{\circ} \mathrm{C}$. The vapor line (TC08) was the highest, with the first three thermocouples in the condenser (TC09, TC10, TC11) decreasing slightly. The vapor became saturated within the condenser, and condensation formed on the interior walls of the tubing. From the point at which the quality of the working fluid was $x=1$ (saturated vapor) to where it reached $x=0$ (saturated liquid), the temperature should have been constant, except for the fact that the pressure dropped slightly due to viscous losses. This drop in the saturation pressure in turn decreased the saturation temperature. Past TC11, the other condenser temperatures (TC12 and TC13) dropped significantly. This showed that the $2 \varphi-1 \varphi$ point, where $x=0$, occurred between TC11 and TC12. The working fluid after this point became a subcooled liquid, where the temperature drop was due to sensible heat extraction by the cold plates. Interestingly, at $t=0$, the temperature at the bayonet inlet (TC14) was higher than the outlet of the condenser. Under typical operation, this was not the case due to convective losses from the liquid lines.

As time progressed from $t=0$ (Fig. 6a), several things occurred nearly simultaneously. The evaporator thermocouple nearest to the vapor manifold (TC07) suddenly decreased, which indicated movement of subcooled liquid from the exit of the bayonet tube into the evaporator. The junction between the evaporator and the compensation chamber (TC15) increased and then decreased in temperature over a relatively short period. This was due to warm liquid in the evaporator section being pushed through the mesh in the secondary wick into the compensation chamber, followed by cooler liquid from the bayonet tube exit. The inlet of the bayonet tube (TC14) decreased, and the two thermocouples measuring the subcooled liquid in the condenser increased (TC12 and TC13). Again, this was indicative of movement of the slug of liquid that existed from the $2 \varphi-1 \varphi$ point in the condenser to the meniscus within the grooves of the secondary wick inside the evaporator section, as shown in Fig. 1b. The dramatic temperature increase in the condenser section (TC12) shows that the $2 \varphi-1 \varphi$ point moved from between TC11 and TC12, across the TC12 location, and then between TC12 and TC13 as shown schematically in Fig. 6b. In fact, TC12 increased to the saturated vapor temperature existing within the first half of the condenser. 
At approximately $t=80 \mathrm{~s}$, the temperatures in the evaporator and the bayonet tube inlet (TC14) started to increase, while the condenser temperatures TC12 and TC13 decreased. This behavior indicated that the liquid slug had reversed direction; i.e., the $2 \varphi-1 \varphi$ point re-crossed thermocouple location TC12 in the condenser. The significant rise in the bayonet inlet temperature TC14 shows that warm liquid originally in the evaporator was now flooding back through the bayonet tube into the liquid line. This movement of liquid out of the evaporator may be due to the sudden appearance of a vapor bubble within the wick structure of the evaporator section which would tend to drive the heated liquid in the evaporator in the opposite direction. As can be seen in Fig. 6a, the period of the oscillation was approximately $150 \mathrm{~s}$. Discussion of flow reversal within LHPs in the literature was limited to operation during startup and shutdown. Douglas et al. [15] discussed flow reversal in LHPs as a phenomenon that occurred during startup and continued until the capillary pressure in the secondary wick could no longer maintain the system pressure drop. Cimbala et al. [16] used neutron radiography to visualize LHP operation and observed flow reversal only occurred when the heat input was reduced to $Q_{\text {in }}=0 \mathrm{~W}$. It was concluded by Cimbala et al. that with no heat input, convective and radiative heat transfer from the LHP to the ambient caused the flow reversal. In general, flow reversal was not discussed as part of normal operation. However, in the present experiment, flow reversal was found at some operating points.

The various steady state LHP temperatures versus transported heat for the stationary case are shown in Fig. 7. The four evaporator temperatures in Fig. 7a increased monotonically with heat transported, but diverged from the vapor outlet temperature. The behavior of the condenser temperatures with heat transported was slightly different, as shown in Fig. 7b. At the lowest heat input value $\left(Q_{\text {in }}=100 \mathrm{~W}\right)$, a significant temperature drop was present between the inlet of the condenser (TC09) and the thermocouples within the condenser. This shows that the $2 \varphi-1 \varphi$ point resided between TC09 and TC10, which means that very little of the available condenser was being used for twophase condensation. This condition also shows that the liquid returning to the evaporator section (TC14) was highly subcooled. As the heat input increased to $Q_{\text {in }}=200 \mathrm{~W}$, the temperatures measured at TC10 and TC11 rose to match that at TC09, which means that the time averaged location of the $2 \varphi-1 \varphi$ point moved farther into the condenser (between TC11 and TC12). At a heat input of $Q_{\text {in }}=300 \mathrm{~W}$, the $2 \varphi-1 \varphi$ point traveled past the end of the condenser into the liquid lines such that the condenser temperature was nearly constant. As the heat input increased, the condenser temperatures continued to rise. However, the average evaporator temperature increased at a faster rate, which is indicative of an increased superheat penalty. 
The thermal performance of the stationary LHP for heat inputs ranging from $Q_{\text {in }}=100$ to $600 \mathrm{~W}$ is shown in Fig. 8. The evaporative heat transfer coefficient, Fig. 8a, decreased monotonically with transported heat. This behavior was controlled by the slope of the average evaporator temperature versus that of the evaporator outlet, as shown in Fig. 7a. The temperature difference $\left(\bar{T}_{\mathrm{e}}-T_{\mathrm{v}}\right)$ defined in Eq. (2) increased more rapidly than $Q_{\text {out }}$, which resulted in an overall decrease in $\bar{h}$. As dry-out was approached, more of the wick in the evaporator section was depleted of liquid, which tended to increase the evaporator temperature. The thermal resistance of the stationary LHP versus heat transported is presented in Fig. 8b, where it is seen to decrease, reach a minimum, and then increase. At low power inputs, the relatively large temperature drop defined by Eq. (3), $\left(\bar{T}_{\mathrm{e}}-\bar{T}_{\mathrm{cp}}\right)$, drove the thermal resistance to a high value. This temperature drop was a result of the fact that most of the condenser section was flooded by subcooled liquid which was close to the cold plate temperature. As the $2 \varphi-1 \varphi$ point moved through and then exited the condenser, the temperature drop decreased with transported heat, which decreased the thermal resistance. The minimum $R$ corresponds to the point in Fig. $7 \mathrm{~b}$ where the $2 \varphi-1 \varphi$ point just exited the condenser. Past this point, the evaporator section increased in temperature more rapidly than the condenser section, which resulted in the thermal resistance increasing with transported heat. The evaporator wall superheat, defined as the difference between the average evaporator temperature and the temperature of the evaporator/compensation chamber junction, was found to monotonically increase with an increasing amount of transported heat, as shown in Fig. 8c. With respect to the evaporative heat transfer coefficient, thermal resistance, and evaporator wall superheat, no notable difference was observed between starting the LHP while the unit was at ambient temperature versus a step change in the evaporator heat input from a lower to higher value or a higher to lower value [13].

The operating characteristics and performance of the stationary LHP for an evaporator heat input of $Q_{\text {in }}=500 \mathrm{~W}$ while varying the compensation chamber heat input from $Q_{\mathrm{cc}}=0$ to $50 \mathrm{~W}$ are shown in Fig. 9. For this particular test, the LHP was allowed to achieve steady state conditions for the given evaporator heat input, after which the compensation chamber heat input was incremented in steps of $5 \mathrm{~W}$, achieving steady state conditions at each step. In Fig. $9 \mathrm{a}$ and $\mathrm{b}$, for $Q_{\mathrm{cc}}=0 \mathrm{~W}$, the evaporator temperatures were relatively uniform, where the vapor exiting the evaporator was slightly superheated and the $2 \varphi-1 \varphi$ point was out of the condenser. When a small amount of heat was input to the system through the compensation chamber $\left(Q_{\mathrm{cc}}=5 \mathrm{~W}\right)$, the evaporator temperatures and the evaporator exit temperature both decreased while the condenser temperatures remained constant. This trend continued until approximately $Q_{\mathrm{cc}}=15 \mathrm{~W}$, at which point the evaporator temperature leveled off, the evaporator exit 
temperature decreased to the saturation temperature within the condenser, and the condenser outlet temperature dropped below the saturation temperature. The decrease in the average evaporator temperature significantly affected the evaporative heat transfer coefficient and the thermal resistance, as shown in Fig. 9c. In fact, $\bar{h}$ increased by $68 \%$ with an increase in the overall heat input of only $3 \%$. The drop in the condenser outlet temperature indicated that the $2 \varphi-1 \varphi$ point moved from the liquid line into the condenser section. For $Q_{\mathrm{cc}} \geq 20 \mathrm{~W}$, the evaporator temperatures increased and the $2 \varphi-1 \varphi$ point continued to move toward the evaporator within the condenser. The increase in the amount of subcooling increased the thermal resistance and decreased the evaporative heat transfer coefficient by way of an increased superheat penalty. This did not hold true for $Q_{\mathrm{cc}}<20 \mathrm{~W}$. When the LHP operated at $Q_{\text {in }}=500$ $\mathrm{W}$, the $2 \varphi-1 \varphi$ interface was located in the liquid return line. Increasing the heat input to the compensation chamber moved the $2 \varphi-1 \varphi$ interface to the condenser outlet at $Q_{\mathrm{cc}}=15 \mathrm{~W}$. Operation at this point maximized the amount of heat transfer due to condensation with the added benefit of cooler liquid in the compensation chamber and evaporator which decreased the thermal resistance and increased the evaporative heat transfer coefficient. By comparison, $\mathrm{Ku}$ [17] indicated that operating the compensation chamber at a higher temperature by using an external heater in effect increases the amount of subcooling in the condenser and liquid return line. This subcooling is necessary to balance the additional heat input and results in underutilizing the condenser and a degradation of the thermal conductance. It is clear that for the present experiment this conclusion was only valid for $Q_{\mathrm{cc}} \geq 20 \mathrm{~W}$, while for $Q_{\mathrm{cc}}<20 \mathrm{~W}$ an increase in LHP performance was observed.

Also of interest is the temperature increase at the bayonet inlet (TC14) starting when $Q_{\mathrm{cc}}=35 \mathrm{~W}$ as seen in Fig. 9b. Transient temperature traces in the condenser, bayonet tube, and evaporator/compensation chamber junction for $Q_{\mathrm{cc}}=25$ to $50 \mathrm{~W}$ are shown in Fig. 10. In Fig. 10a and b, with $Q_{\mathrm{cc}}=25$ and $30 \mathrm{~W}$, subcooled liquid moved forward through the bayonet inlet as seen in typical operation. In Fig. 10c through Fig. 10f, with $Q_{\mathrm{cc}}=35$ through $50 \mathrm{~W}$, a sudden increase in temperature at the bayonet inlet (TC14) higher than the condenser outlet (TC13) showed that flow reversal occurred in the evaporator section. This was similar to the oscillating phenomena described for $Q_{\text {in }}=$ $200 \mathrm{~W}$ except that the liquid-vapor meniscus in the secondary wick was driven backward by the elevated vapor pressure within the compensation chamber, which was due to the heat input at the shell of the compensation chamber. In addition, the temperature of the evaporator/compensation chamber interface (TC15) did not vary appreciably, which was different than that seen at $Q_{\mathrm{in}}=200 \mathrm{~W}$ and $Q_{\mathrm{cc}}=0 \mathrm{~W}$. The liquid-vapor meniscus moved backward due to the increased pressure within the compensation chamber until a point at which the pressure was 
balanced. Forward flow then resumed and heat was lost through the liquid line, shown by the slow decrease in temperature at the bayonet inlet (TC14) to the temperature of the subcooled liquid at the condenser outlet (TC13).

To further explore compensation chamber heat input and heat loss to the ambient, Table 4 shows the effect of operating the stationary LHP for $Q_{\text {in }}=500 \mathrm{~W}$ with the compensation chamber uninsulated, insulated, temperature controlled to $T_{\mathrm{cc}}=72.8^{\circ} \mathrm{C}$ via simultaneous heat input to the compensation chamber $\left(Q_{\mathrm{cc}}=20 \mathrm{~W}\right)$ and evaporator, and preconditioning the temperature to $T_{\mathrm{cc}}=72.8^{\circ} \mathrm{C}$ via heat input $\left(Q_{\mathrm{cc}}=100 \mathrm{~W}\right.$ reduced to $\left.Q_{\mathrm{cc}}=20 \mathrm{~W}\right)$ prior to heat input to the evaporator. For this series of tests, thermocouple TC15 was relocated to the top side of the compensation chamber to directly monitor its operating temperature. It was observed that the average evaporator temperature increased, the evaporative heat transfer coefficient decreased, and the thermal resistance increased in the uninsulated state when compared to the insulated case. The uninsulated compensation chamber operated at a temperature $3.6^{\circ} \mathrm{C}$ lower than the insulated case as expected due to free convection and radiative heat loss. To estimate the amount of heat loss to the ambient in the uninsulated case, the exposed compensation chamber was modeled as a cylinder in free convection with radiation. The average Nusselt number for free convection was given by [18]

$$
\overline{\mathrm{Nu}}_{\mathrm{D}}=\left\{0.60+\frac{0.387 \mathrm{Ra}_{\mathrm{D}}^{1 / 6}}{\left[1+(0.559 / \mathrm{Pr})^{9 / 16}\right]^{8 / 27}}\right\}^{2}
$$

where

$$
\operatorname{Ra}_{\mathrm{D}}=\frac{g \beta\left(T_{\mathrm{s}}-T_{\infty}\right) D^{3}}{v \alpha}
$$

with air properties evaluated at the average temperature of the freestream and the surface. The average heat transfer coefficient was given by

$$
\bar{h}=\frac{k}{D} \overline{\mathrm{Nu}}_{\mathrm{D}}
$$

The total heat loss per unit length from the compensation chamber was given by

$$
q_{\mathrm{tot}}^{\prime}=q_{\mathrm{conv}}^{\prime}+q_{\mathrm{rad}}^{\prime}=\bar{h} \pi D\left(T_{\mathrm{s}}-T_{\infty}\right)+\epsilon \pi D \sigma\left(T_{\mathrm{s}}^{4}-T_{\mathrm{surr}}^{4}\right)
$$

The emissivity of grade 2 titanium used in this calculation was $\varepsilon=0.3$ [19]. The heat loss from the compensation chamber for the uninsulated case was found to be $Q_{\mathrm{cc}}=-6.2 \mathrm{~W}$. When the uninsulated case was included with the previous compensation chamber heat input data, it was found that evaporative heat transfer coefficient and thermal 
resistance followed the trends shown in Fig. 9(c). These results were expected since removing the insulation from the compensation chamber in effect provided additional cooling, which moved the $2 \varphi-1 \varphi$ point away from the condenser. The average evaporator and cold plate temperatures were significantly different which was likely due to a $10^{\circ} \mathrm{C}$ higher ambient temperature during the test involving the variation of compensation chamber heat input from $Q_{\mathrm{cc}}=0$ to $50 \mathrm{~W}$. As a result, for this particular case, it was advantageous to operate the LHP compensation chamber insulated for improved performance. For controlling the temperature of the compensation chamber, the evaporative heat transfer coefficient, thermal resistance, and operating temperatures were nearly identical between simultaneous compensation chamber and evaporator heat input startup and compensation chamber temperature preconditioning, demonstrating that the startup procedure had no impact on steady state conditions. However, preconditioning the compensation chamber required approximately one hour less time to reach steady state conditions over the simultaneous heat input startup.

Transient LHP temperatures for a typical test at elevated acceleration $\left(a_{\mathrm{z}}=1.0 \mathrm{~g}, a_{\mathrm{r}}>0 \mathrm{~g}\right)$ are presented in Fig. 11. With the LHP at ambient conditions, the recirculating constant-temperature bath in the low temperature loop was set to $T_{\text {eg }}=35^{\circ} \mathrm{C}$. Heat was applied as a step function (in this case, $Q_{\text {in }}=600 \mathrm{~W}$ ) while simultaneously starting the pump for the high-temperature loop $\left(\dot{m}_{\mathrm{cp}}=0.0077 \mathrm{~kg} / \mathrm{s}\right)$. In addition, the radial acceleration was increased to $a_{\mathrm{r}}=$ $0.1 \mathrm{~g}$, which was a nominally small value to prevent damage to the power slip rings (Fig. 11a). The LHP was allowed to achieve steady state conditions at $a_{\mathrm{r}}=0.1 \mathrm{~g}$, indicated by $\mathrm{d} T / \mathrm{d} t$ (Fig. 11c) decreasing to below the threshold of $0.01 \mathrm{~K} / \mathrm{min}$, then the acceleration was increased to the next desired radial acceleration value (in this case, $a_{\mathrm{r}}=10.0 \mathrm{~g}$ ). The LHP was again allowed to achieve steady state conditions at the given acceleration (Fig. 11b), then the acceleration was reduced back to $a_{\mathrm{r}}=0.1 \mathrm{~g}$ for a minimum of thirty minutes. If another elevated acceleration was desired, steady state at $a_{\mathrm{r}}=0.1 \mathrm{~g}$ was reached before increasing the acceleration level. When the acceleration was increased to $a_{\mathrm{r}}=10.0 \mathrm{~g}$ at $t=15,000 \mathrm{~s}$ in Fig. $11 \mathrm{~b}$, the average evaporator temperature increased by $11^{\circ} \mathrm{C}$. The $2 \varphi-1 \varphi$ point moved to the condenser outlet from the liquid line with increasing acceleration, indicated by the small oscillations in temperature at the TC13. The amount of subcooling increased overall as indicated by the decrease in temperature at the bayonet inlet (TC14). These phenomena may be due in part to fluid redistribution in the LHP and are discussed in the following paragraph.

As the rotational velocity of the centrifuge table increased, the resultant acceleration vector magnitude and direction changed (Fig. 12a), which influenced the distribution of fluid in the LHP. Subcooled liquid entering the 
primary wick of the evaporator was forced to the outboard side of the evaporator body, opposite of the heat source, and perhaps led to a partial dry-out of the wick (Fig. 12b). The elevated acceleration also hindered the ability of the secondary wick in the compensation chamber to supply the evaporator with liquid due to pooling. In the condenser, pooling occurred in the bends of the condenser coil, again due to the acceleration gradient. Depending on the acceleration vector direction, this pooling could either open or close the passage to vapor flow (Fig. 12c). All of these phenomena are a result of centrifuge testing. Due to the short radius, strong acceleration gradients occur that could have advantageous or adverse effects on the LHP operation. Operation in an aircraft environment, with significantly larger radii during turns, may provide a more uniform acceleration gradient across the LHP and potentially yield different temperature profiles, evaporative heat transfer coefficients, and thermal resistances.

The thermal performance of the LHP for radial accelerations ranging from $a_{\mathrm{r}}=0.0$ to $10.0 \mathrm{~g}$ and heat inputs ranging from $Q_{\text {in }}=100$ to $600 \mathrm{~W}$ is shown in Fig. 13. The evaporative heat transfer coefficient, Fig. 13a, again decreased with transported heat, similar to the trend in Fig. 8a for the stationary LHP. The thermal resistance of the LHP (Fig. 13b), was found to decrease to a minimum and then increase, again similar to the stationary test results shown in Fig. 8b. In fact, when combining the stationary and elevated acceleration test data, it was found that the evaporative heat transfer coefficient and thermal resistance data were in close agreement with each other, regardless of the radial acceleration. This indicated that bench top testing of the LHP is a reliable method for determining the evaporative heat transfer coefficient and thermal resistance of a LHP in an elevated acceleration environment. However, it will be shown that this was not true with respect to finding the dry-out limit. The wall superheat (Fig. 13c) was higher at elevated accelerations when compared to $a_{\mathrm{r}}=0 \mathrm{~g}$. This was possibly due to fluid redistribution in the evaporator forcing liquid away from the heater. The dependence of $\Delta T_{\mathrm{sh}}$ on both $Q_{\text {out }}$ and $a_{\mathrm{r}}$ found in the current research is different than the results of $\mathrm{Ku}$ et al. [4], who found no significant variation of the wall superheat over the ranges of $Q_{\text {out }}$ and $a_{\mathrm{r}}$ tested.

$\mathrm{Ku}$ et al. [4][5] observed that radial acceleration changed the fluid distribution throughout the LHP which changed operating temperatures and that acceleration could either increase or decrease LHP operating temperatures. In addition, evaporator temperature overshoots were observed for mid-range heat inputs, and the evaporator wall superheat was independent of heat input and acceleration during startup. In the present elevated acceleration tests, it was observed that the accelerating force changed the fluid distribution within the LHP, causing the operating temperatures to change. However, in all instances, it was observed that elevated acceleration forces increased 
operating temperatures over those at $a_{\mathrm{r}}=0.1 \mathrm{~g}$. Evaporator temperature overshoots were not observed in any of the elevated acceleration tests.

The transient response of the LHP during a series of dry-out events is shown in Fig. 14. Dry-out was indicated by a steady increase in the evaporator temperature and a decrease in the heat extracted by the calorimeter $Q_{\text {out }}$. In addition, the position of the $2 \varphi-1 \varphi$ point in the condenser moved toward the evaporator as indicated by a sequential decrease in the condenser temperatures. This occurred because the evaporator no longer generated a sufficient flow of vapor which changed the operating point of the LHP. In Fig. 14a, the LHP reached steady state while rotating slowly at $a_{\mathrm{r}}=0.1 \mathrm{~g}$ and $Q_{\mathrm{in}}=400 \mathrm{~W}$. The rotational speed of the centrifuge table was increased until the radial acceleration reached $a_{\mathrm{r}}=8.0 \mathrm{~g}$ at $t=300 \mathrm{~s}$. After the evaporator temperature TC06 reached $T_{\mathrm{e}, \max }=150^{\circ} \mathrm{C}$, the radial acceleration was reduced back to $a_{\mathrm{r}}=0.1 \mathrm{~g}$. At this time, the evaporator temperature continued to increase, but then leveled off and then decreased back to nearly the same temperature as the previous steady state. In fact, all of the LHP temperatures returned to within $1{ }^{\circ} \mathrm{C}$ of the original steady state except for TC13 (condenser outlet), which returned to within $4{ }^{\circ} \mathrm{C}$ of the previous steady state. The higher temperature at TC13 was attributable to a slight change in the location of the $2 \varphi-1 \varphi$ point in the condenser. This recovery behavior shows that the LHP was capable of repriming at the end of an acceleration burst even if the heat input remained constant. In Fig. 14b and c, the radial acceleration was again increased from $a_{\mathrm{r}}=0.1$ to $8.0 \mathrm{~g}$ with the same heat input $\left(Q_{\text {in }}=400 \mathrm{~W}\right)$. In fact, all of the experiments presented in Fig. 14 were performed sequentially. In Fig. 14b, the evaporator temperature TC06 was allowed to reach $T_{\mathrm{e}, \max }=175^{\circ} \mathrm{C}$ before decreasing the radial acceleration to $a_{\mathrm{r}}=0.1 \mathrm{~g}$, and in Fig. $14 \mathrm{c}$, the evaporator temperature TC06 reached $T_{\mathrm{e}, \max }=200^{\circ} \mathrm{C}$ before decelerating. In each instance, the evaporator temperature continued to increase, reached a maximum, and then decreased to the original steady state. However, the intensity of dry-out did seem to have an impact on the ability of the LHP to reprime. In Fig. 14c, with a maximum evaporator temperature at deceleration of $T_{\mathrm{e}, \max }=200^{\circ} \mathrm{C}$, the evaporator temperature reached two maximums before finally decreasing back to the previous steady state, whereas in Fig. 14a and Fig. 14b, the maximum evaporator temperatures reached a peak and then monotonically decreased. This indicated that if the evaporator temperatures were much higher than $200^{\circ} \mathrm{C}$, the LHP may not have recovered, which would have required that the heat input be reduced to zero.

The temperature traces associated with the test at $Q_{\mathrm{in}}=200 \mathrm{~W}$ and $a_{\mathrm{r}}=0.1$ and $a_{\mathrm{r}}=4.0 \mathrm{~g}$ are shown in Fig. 15 . Following the previously mentioned startup procedures, the LHP reached a quasi-steady state while the centrifuge 
table rotated slowly for $a_{\mathrm{r}}=0.1 \mathrm{~g}$, as shown in Fig. 15a. Similar to the stationary case at this heat input, the LHP temperatures oscillated, showing that the heat pipe was operating during reversals in the liquid flow due to the liquid-vapor meniscus in the secondary wick moving back and forth. Overall, the temperatures shown in Fig. 15a were quite close to the case shown in Fig. $6 \mathrm{a}$. In addition, the period of the oscillation of the $a_{\mathrm{r}}=0.1 \mathrm{~g}$ case was nearly identical to the $a_{\mathrm{r}}=0 \mathrm{~g}$ case (approximately $175 \mathrm{~s}$ ). The only significant differences in the independent variables between the two tests were the ambient temperature $\left(\Delta T_{\mathrm{amb}}=5.3^{\circ} \mathrm{C}\right)$, and the relatively small value of the radial acceleration. Of note, however, was the location of the $2 \varphi-1 \varphi$ point in the condenser: For $a_{\mathrm{r}}=0 \mathrm{~g}$, this point resided close to TC12, whereas for the case in which $a_{\mathrm{r}}=0.1 \mathrm{~g}$, the $2 \varphi-1 \varphi$ point was near TC10. The linear distance between these two points was approximately $143 \mathrm{~cm}$. While it was impossible to know the exact location of the $2 \varphi$ $1 \varphi$ point due to the coarse resolution of the thermocouples in the condenser, it was obvious that the location had changed significantly between the two cases.

After achieving the quasi-steady state at $a_{\mathrm{r}}=0.1 \mathrm{~g}$, the radial acceleration was increased to $a_{\mathrm{r}}=4.0 \mathrm{~g}$, and the LHP again reached a quasi-steady state, as shown in Fig. 15b. The average evaporator temperature increased by more than $30^{\circ} \mathrm{C}$, and the temperature in the bayonet inlet ranged from $37 \leq T_{\mathrm{b}} \leq 70^{\circ} \mathrm{C}$, which was a much larger range than that for $a_{\mathrm{r}}=0.1 \mathrm{~g}$. Oscillations were again seen at this acceleration level, but the period of the oscillations increased to approximately $350 \mathrm{~s}$. This may be due to the distance that the meniscus travelled within the evaporator, which resulted in wider swings in the evaporator temperatures and significant oscillations of the cold plate outlet temperature, which was nearly steady in the $a_{\mathrm{r}}=0.1 \mathrm{~g}$ case.

The steady state performance map for the LHP relating radial acceleration and heat transported for $a_{\mathrm{r}}=0.0$ to $10.0 \mathrm{~g}$ and $Q_{\mathrm{in}}=100$ to $600 \mathrm{~W}$ is shown in Fig. 16. It was observed that dry-out conditions occurred at varying radial accelerations for $Q_{\text {in }}=100$ to $400 \mathrm{~W}$. Dry-out conditions were not observed through $a_{\mathrm{r}}=10.0 \mathrm{~g}$ at $Q_{\mathrm{in}}=500$ and 600 W. This was likely due to a combination of a larger fluid inventory in the compensation chamber at higher heat loads and fluid distribution in the condenser. Quasi-steady state conditions were observed at $Q_{\text {in }}=200 \mathrm{~W}$ and $0.0 \leq$ $a_{\mathrm{r}} \leq 4.0 \mathrm{~g}$. This demonstrated that bench-top testing cannot be used to determine the dry-out limit with respect to elevated acceleration. 


\section{Conclusions}

The effect of changes in evaporator heat input, compensation chamber heat input, and radial acceleration on the performance of a titanium-water loop heat pipe were investigated for $Q_{\mathrm{in}}=100$ to $600 \mathrm{~W}, Q_{\mathrm{cc}}=0$ to $50 \mathrm{~W}$, and $a_{\mathrm{r}}=$ 0.0 to $10.0 \mathrm{~g}$. For evaporator heat inputs ranging from $Q_{\text {in }}=100$ to $600 \mathrm{~W}$, it was observed that the evaporative heat transfer coefficient decreased monotonically, the thermal resistance decreased to a minimum and then increased, and the evaporator wall superheat monotonically increased. Flow reversal was observed at $Q_{\text {in }}=200 \mathrm{~W}$ due to vapor bubble generation in the evaporator.

When examining the effect of compensation chamber heat input for $Q_{\text {in }}=500 \mathrm{~W}$, it was found that the average evaporator temperatures dropped by $15^{\circ} \mathrm{C}$ and evaporative heat transfer coefficient improved by $68 \%$ with only a $3 \%$ increase in heat load. These results differ from $\mathrm{Ku}[17]$ in that an improvement was observed for compensation chamber heat input up to the point where subcooling was occurring in the condenser. Periodic flow reversals were observed starting at $Q_{\mathrm{cc}}=35 \mathrm{~W}$ due to the increased pressure in the compensation chamber driving the liquid/vapor meniscus in the secondary wick mesh backward. Operating the LHP compensation chamber uninsulated at $Q_{\text {in }}=500$ W was found to degrade the LHP performance for this particular case and preconditioning the compensation chamber temperature prior to evaporator heat input shortened the time to reach steady state.

When examining the effect of radial acceleration, it was found that dry-out conditions occurred more readily at lower heat inputs $\left(Q_{\text {in }}=100\right.$ to $\left.400 \mathrm{~W}\right)$ than at higher heat inputs $\left(Q_{\text {in }}=500\right.$ to $\left.600 \mathrm{~W}\right)$. The LHP was found to be able to reprime after an acceleration event that caused dry-out without the heat input being reduced to zero. It was also observed that radial acceleration had little effect on the evaporative heat transfer coefficient and thermal resistance of the LHP. The evaporator wall superheat was found to be higher at steady state elevated accelerations when compared to $a_{\mathrm{r}}=0 \mathrm{~g}$. This led to conclusion that bench top testing of the LHP is a reliable method for determining the evaporative heat transfer coefficient and thermal resistance of a LHP in an elevated acceleration environment induced by a centrifuge table, but is not sufficient for determining the dry-out limit or evaporator wall superheat. These results may or may not actually occur in an aircraft environment as centrifuge operation can induce artifacts in the data due to the short radius of operation. 


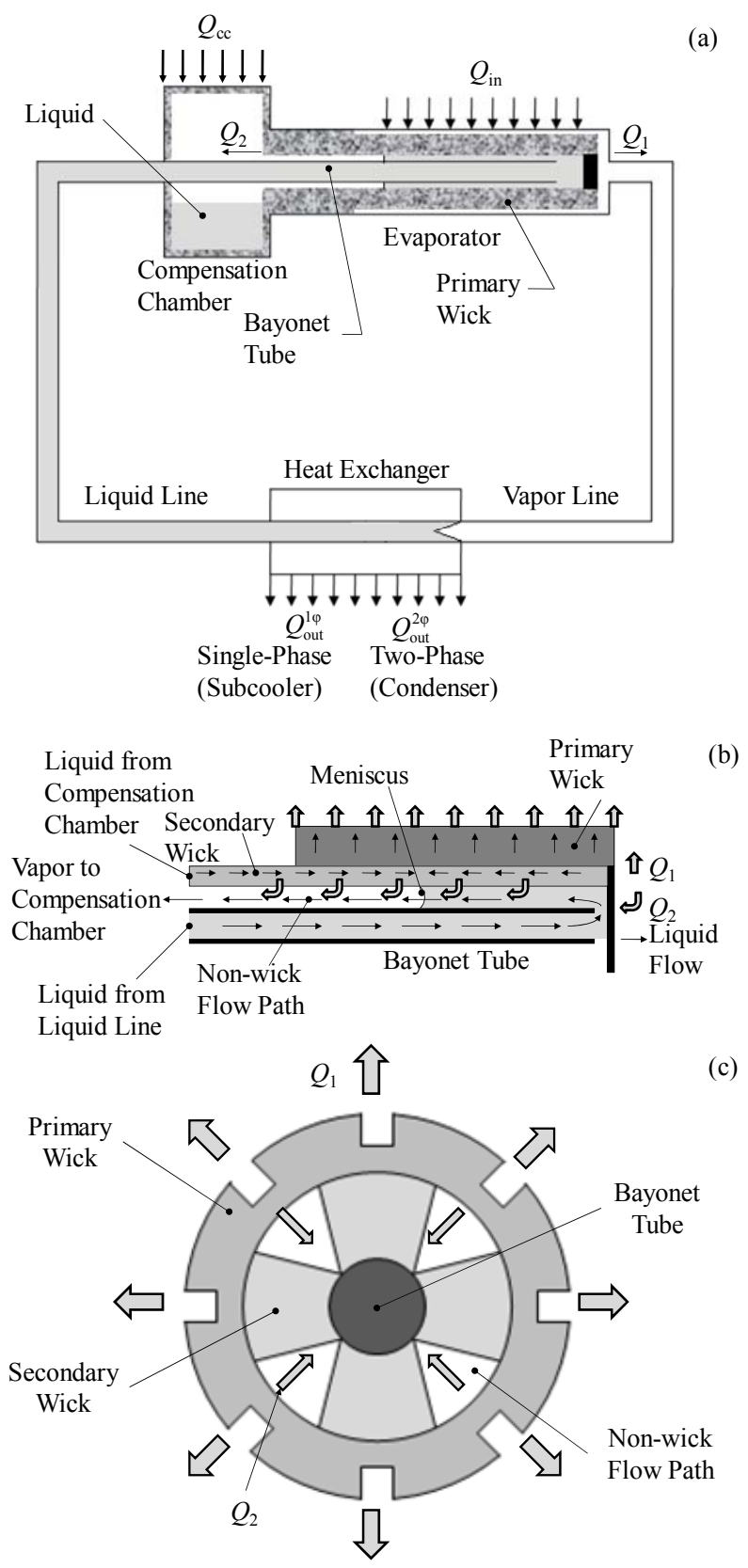

Fig. 1 Loop heat pipe operation: (a) Overall schematic; (b) Side view of evaporator; (c) Cross-sectional view of evaporator. Adapted and reprinted with permission from AIAA [3]. 

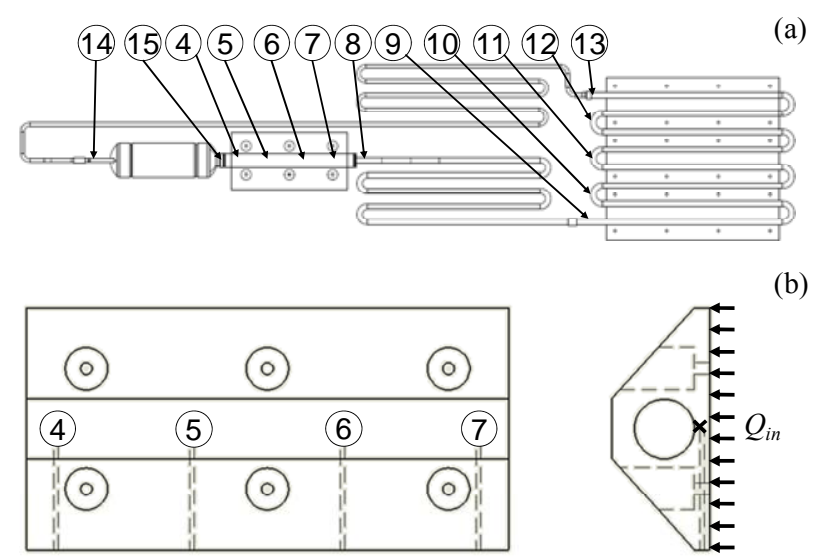

(b)

Fig. 2 Thermocouple locations on the LHP: (a) Locations of thermocouples TC04 through TC15 across the LHP; (b) Locations of TC04 through TC07 within the evaporator. 


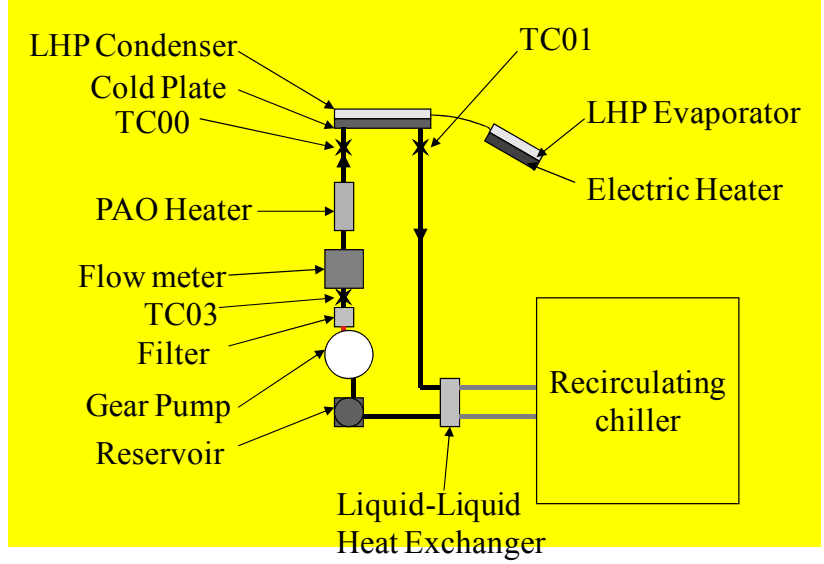

Fig. 3 Experimental setup schematic. 

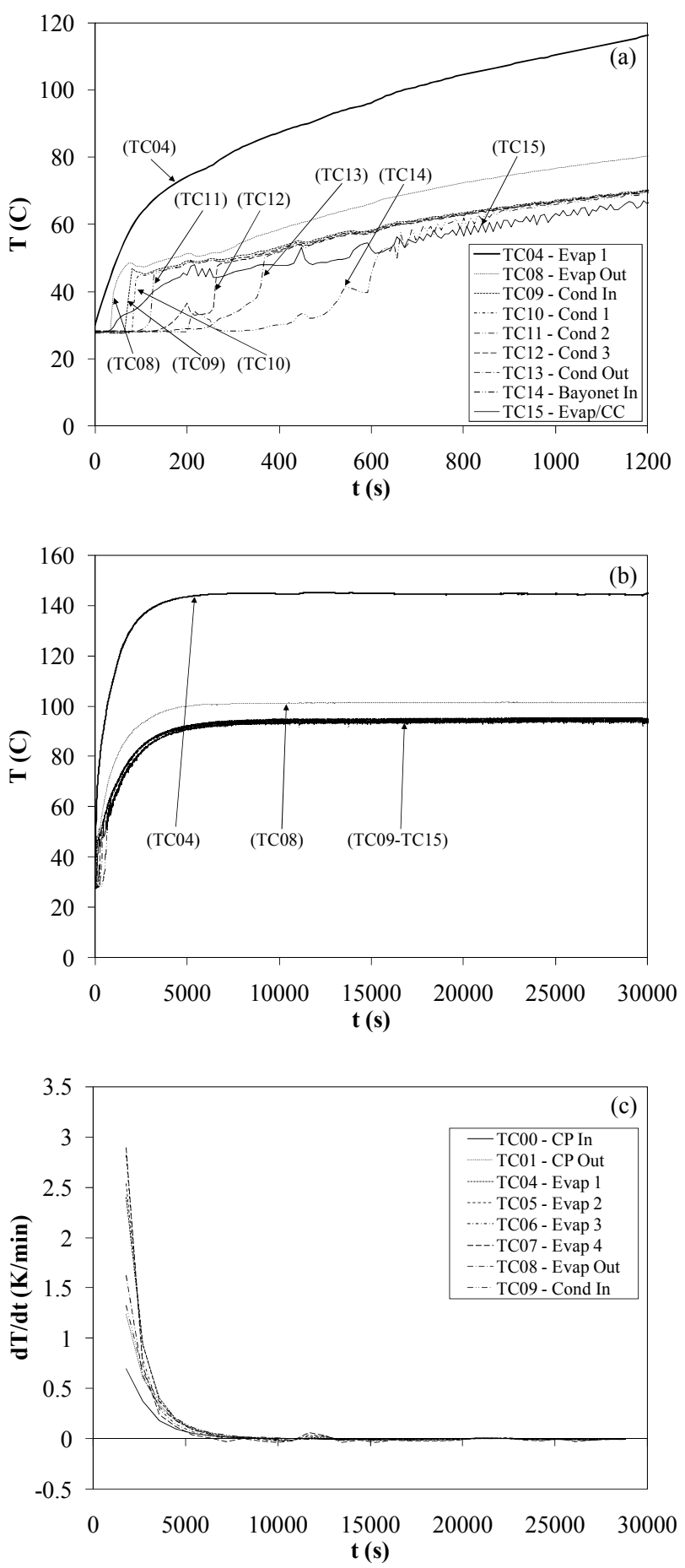

Fig. 4 Transient startup of the stationary LHP $\left(Q_{\text {in }}=600 \mathrm{~W}, Q_{\mathrm{cc}}=0 \mathrm{~W}, a_{\mathrm{r}}=0 \mathrm{~g}, \dot{m}_{\mathrm{cp}}=0.0077 \mathrm{~kg} / \mathrm{s}, \bar{T}_{\mathrm{cp}}=\right.$ $67.7^{\circ} \mathrm{C}, T_{\mathrm{amb}}=38.1^{\circ} \mathrm{C}$ ): (a) Initial startup; (b) Complete startup until steady state; (c) Transient rate of change of temperatures. 

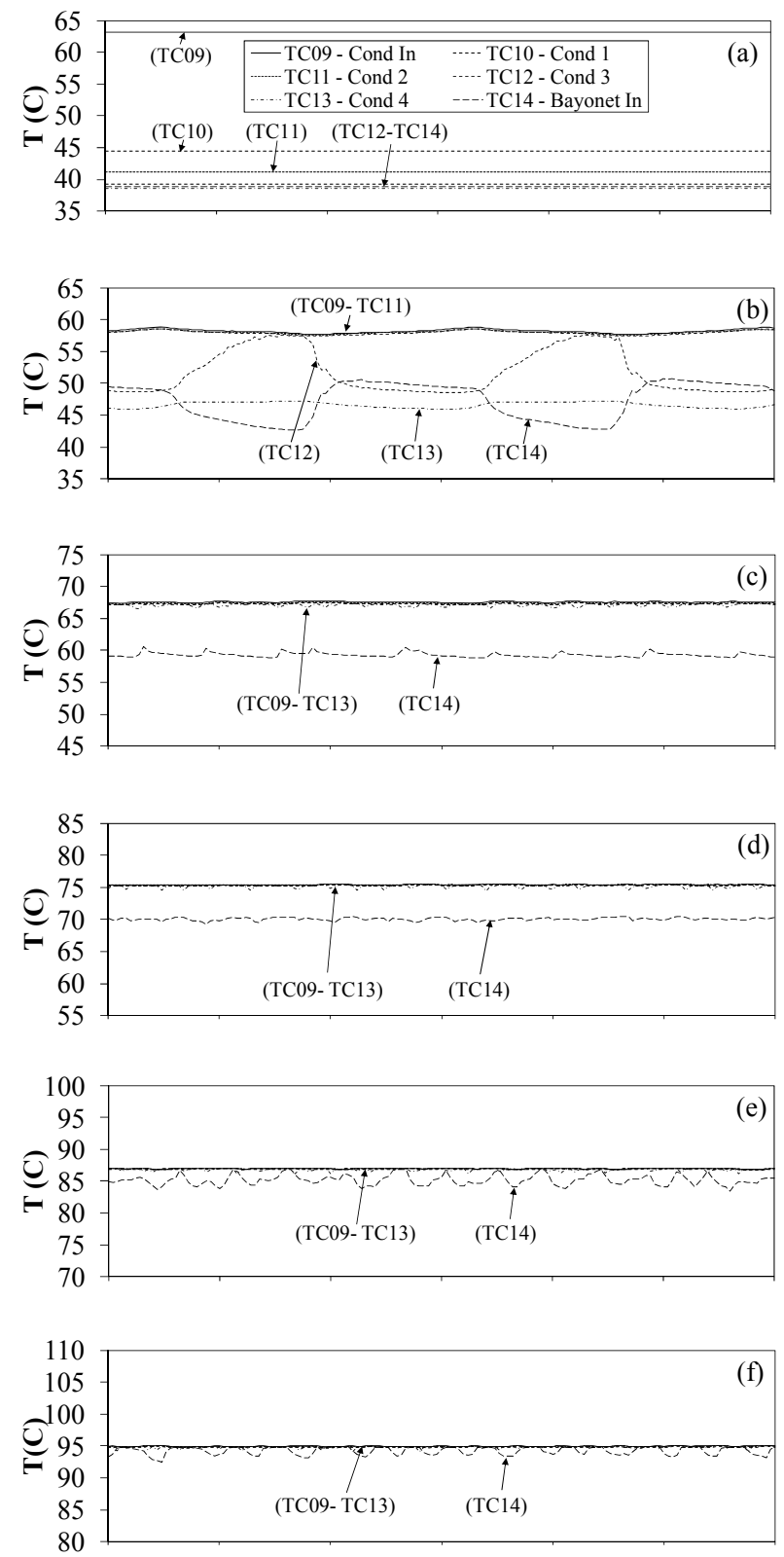

Fig. 5 Temperature traces in the condenser and bayonet tube of the stationary LHP $\left(Q_{\mathrm{cc}}=0 \mathrm{~W}, a_{\mathrm{r}}=0 \mathrm{~g}, \dot{m}_{\mathrm{cp}}\right.$ $=0.0077 \mathrm{~kg} / \mathrm{s}, 36.8 \leq \bar{T}_{\mathrm{cp}} \leq 71.6^{\circ} \mathrm{C}, 31.7 \leq T_{\mathrm{amb}} \leq 38.1^{\circ} \mathrm{C}$ ): (a) $Q_{\text {in }}=100 \mathrm{~W}$; (b) $Q_{\text {in }}=200 \mathrm{~W}$; (c) $Q_{\text {in }}=300 \mathrm{~W}$; (d) $Q_{\text {in }}=400 \mathrm{~W}$; (e) $Q_{\text {in }}=500 \mathrm{~W}$; (f) $Q_{\text {in }}=600 \mathrm{~W}$. 

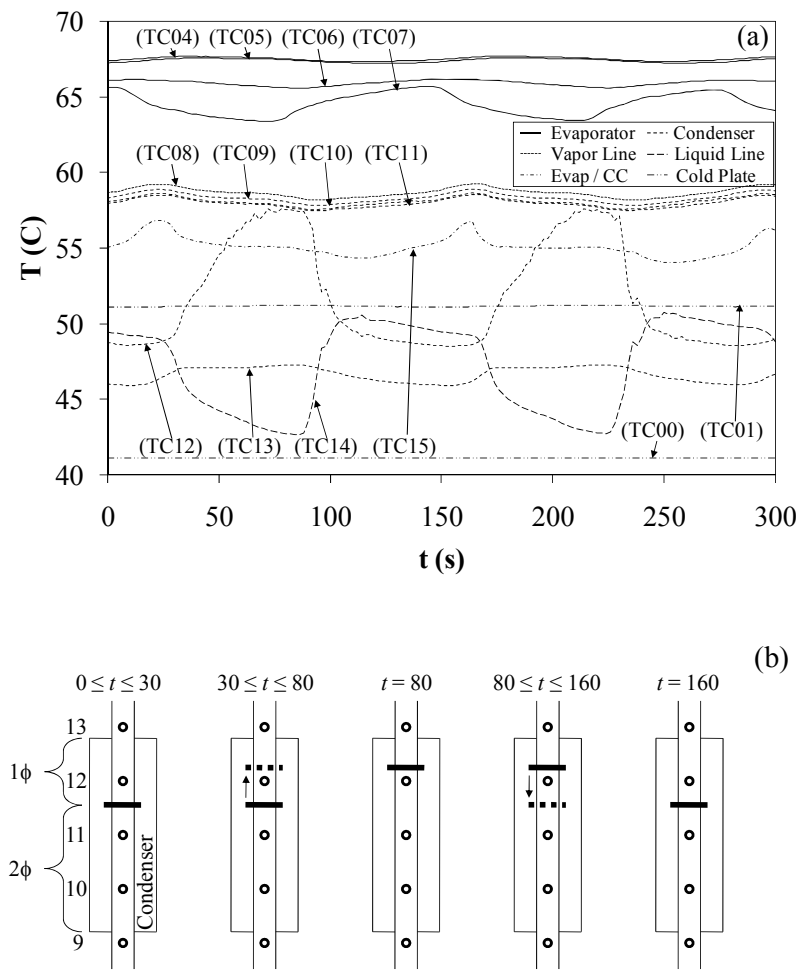

(b)

Fig. 6 Quasi-steady operation of the stationary LHP for $Q_{\mathrm{in}}=200 \mathrm{~W}\left(Q_{\mathrm{cc}}=0 \mathrm{~W}, a_{\mathrm{r}}=0 \mathrm{~g}, \dot{\mathrm{m}}_{\mathrm{cp}}=0.0077 \mathrm{~kg} / \mathrm{s}\right.$, $\bar{T}_{\mathrm{cp}}=46.1^{\circ} \mathrm{C}, T_{\mathrm{amb}}=31.7^{\circ} \mathrm{C}$ ): (a) Temperature traces; (b) $2 \varphi-1 \varphi$ point oscillation in the condenser. 

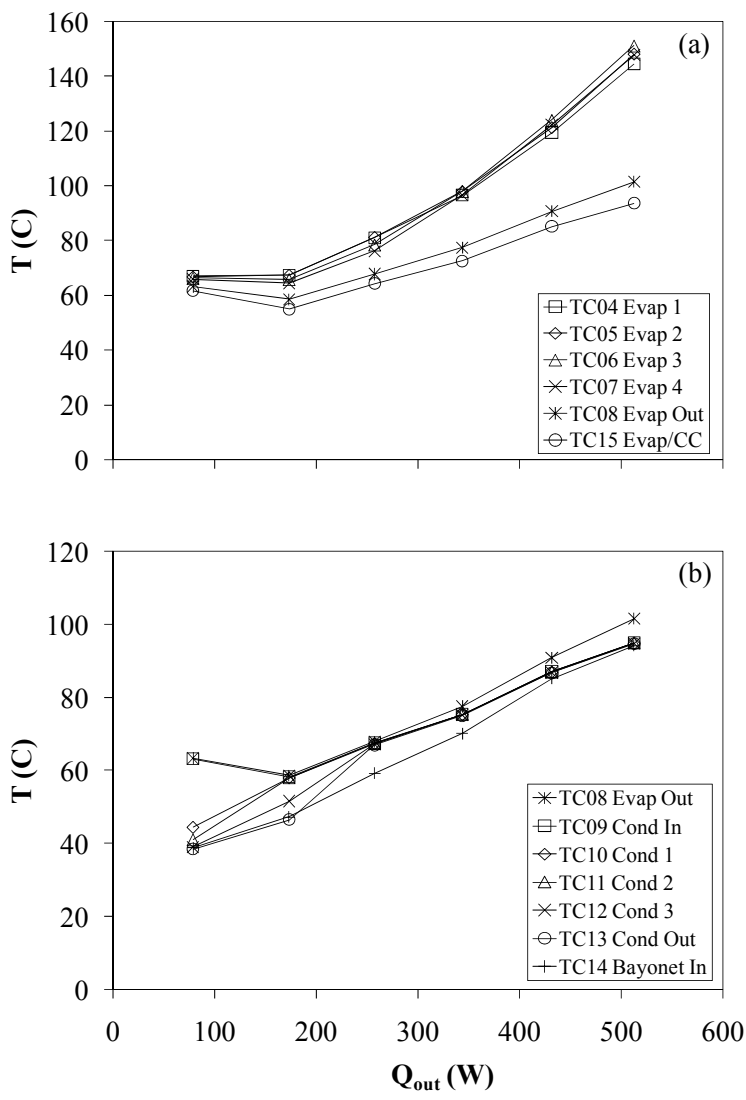

Fig. 7 Steady state temperature distribution versus transported heat for the stationary $L H P\left(Q_{\mathrm{cc}}=0 \mathrm{~W}, a_{\mathrm{r}}=\right.$ $0 \mathrm{~g}, \dot{m}_{\mathrm{cp}}=0.0077 \mathrm{~kg} / \mathrm{s}, 36.8 \leq \bar{T}_{\mathrm{cp}} \leq 71.6^{\circ} \mathrm{C}, 31.7 \leq T_{\mathrm{amb}} \leq 38.1^{\circ} \mathrm{C}$ ): (a) Evaporator section; (b) Condenser section. 

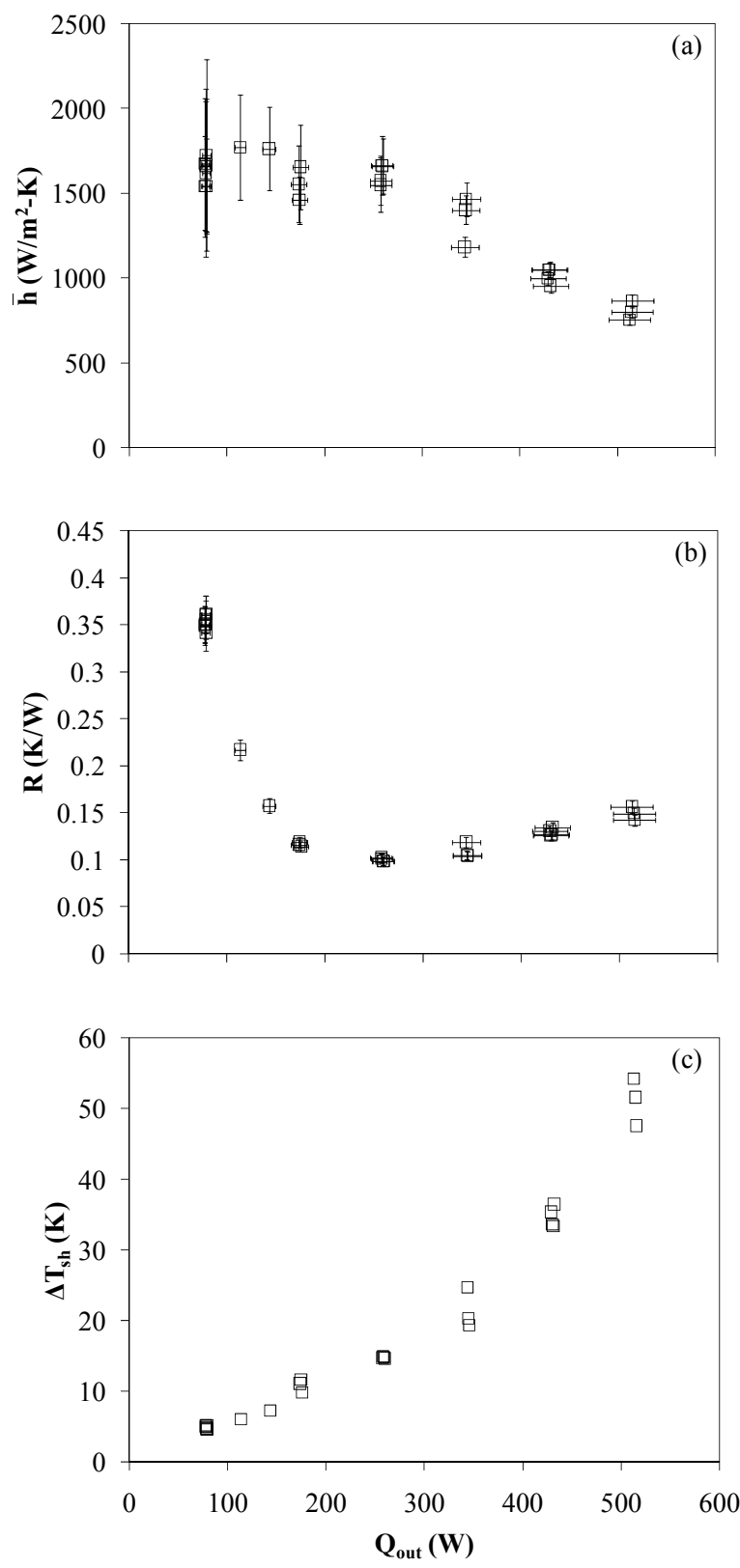

Fig. 8 Steady state performance characteristics of the stationary LHP versus transported heat $\left(Q_{\mathrm{cc}}=0 \mathrm{~W}, a_{\mathrm{r}}\right.$ $=0 \mathrm{~g}, \dot{m}_{\mathrm{cp}}=0.0077 \mathrm{~kg} / \mathrm{s}, 36.8 \leq \bar{T}_{\mathrm{cp}} \leq 67.7^{\circ} \mathrm{C}, 27.6 \leq T_{\mathrm{amb}} \leq 38.7^{\circ} \mathrm{C}$ ): (a) Evaporative heat transfer coefficient;

(b) Thermal resistance; (c) Evaporator wall superheat. 

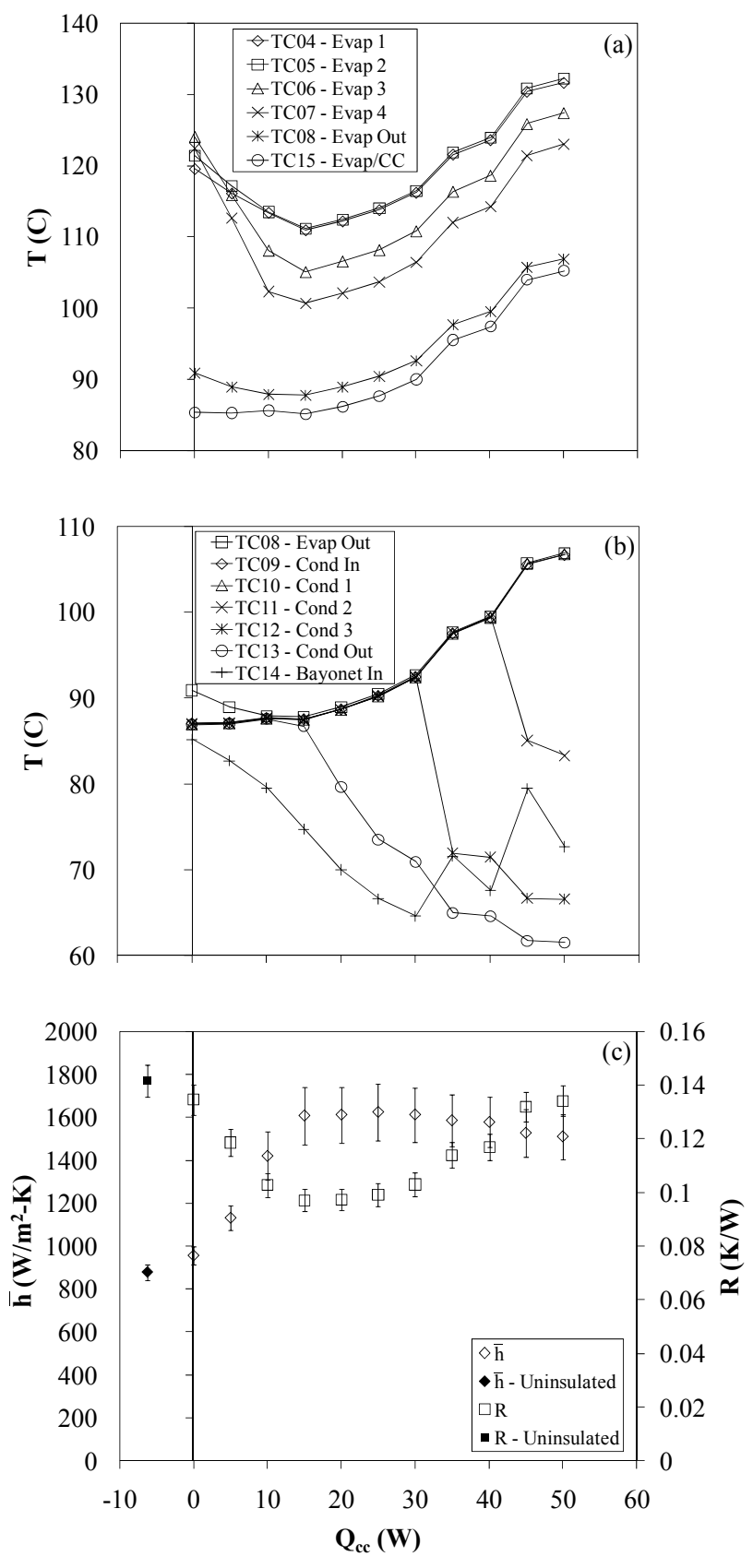

Fig. 9 Steady state performance characteristics of the stationary LHP versus compensation chamber heat input $\left(Q_{\text {in }}=500 \mathrm{~W}, a_{\mathrm{r}}=0 \mathrm{~g}, \dot{m}_{\mathrm{cp}}=0.0077 \mathrm{~kg} / \mathrm{s}, 63.4 \leq \bar{T}_{\mathrm{cp}} \leq 64.8^{\circ} \mathrm{C}, 36.1 \leq T_{\mathrm{amb}} \leq 38.1^{\circ} \mathrm{C}\right)$ : (a) Evaporator temperatures; (b) Condenser temperatures; (c) Evaporative heat transfer coefficient and thermal resistance. 

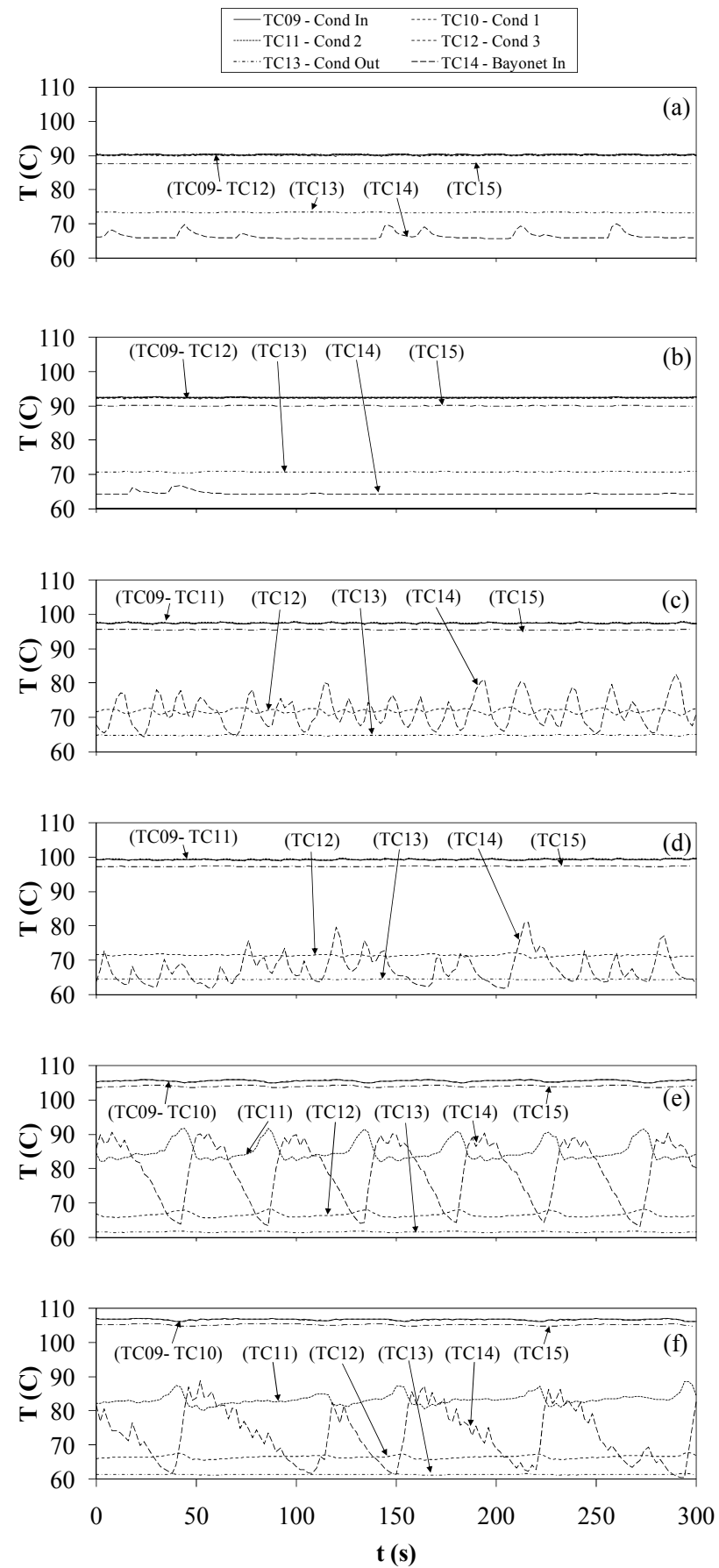

Fig. 10 Temperature traces in the condenser and bayonet tube of the stationary $L H P$ for $Q_{\mathrm{cc}}=25$ to $50 \mathrm{~W}$ $\left(Q_{\mathrm{in}}=500 \mathrm{~W}, a_{\mathrm{r}}=0 \mathrm{~g}, \dot{\mathrm{m}}_{\mathrm{cp}}=0.0077 \mathrm{~kg} / \mathrm{s}, 63.4 \leq \bar{T}_{\mathrm{cp}} \leq 64.8^{\circ} \mathrm{C}, 36.1 \leq T_{\mathrm{amb}} \leq 38.1^{\circ} \mathrm{C}\right):$ (a) $Q_{\mathrm{cc}}=25 \mathrm{~W}$; (b) $Q_{\mathrm{cc}}=$ $30 \mathrm{~W}$; (c) $Q_{\mathrm{cc}}=35 \mathrm{~W}$; (d) $Q_{\mathrm{cc}}=40 \mathrm{~W}$; (e) $Q_{\mathrm{cc}}=45 \mathrm{~W}$; (f) $Q_{\mathrm{cc}}=50 \mathrm{~W}$. 

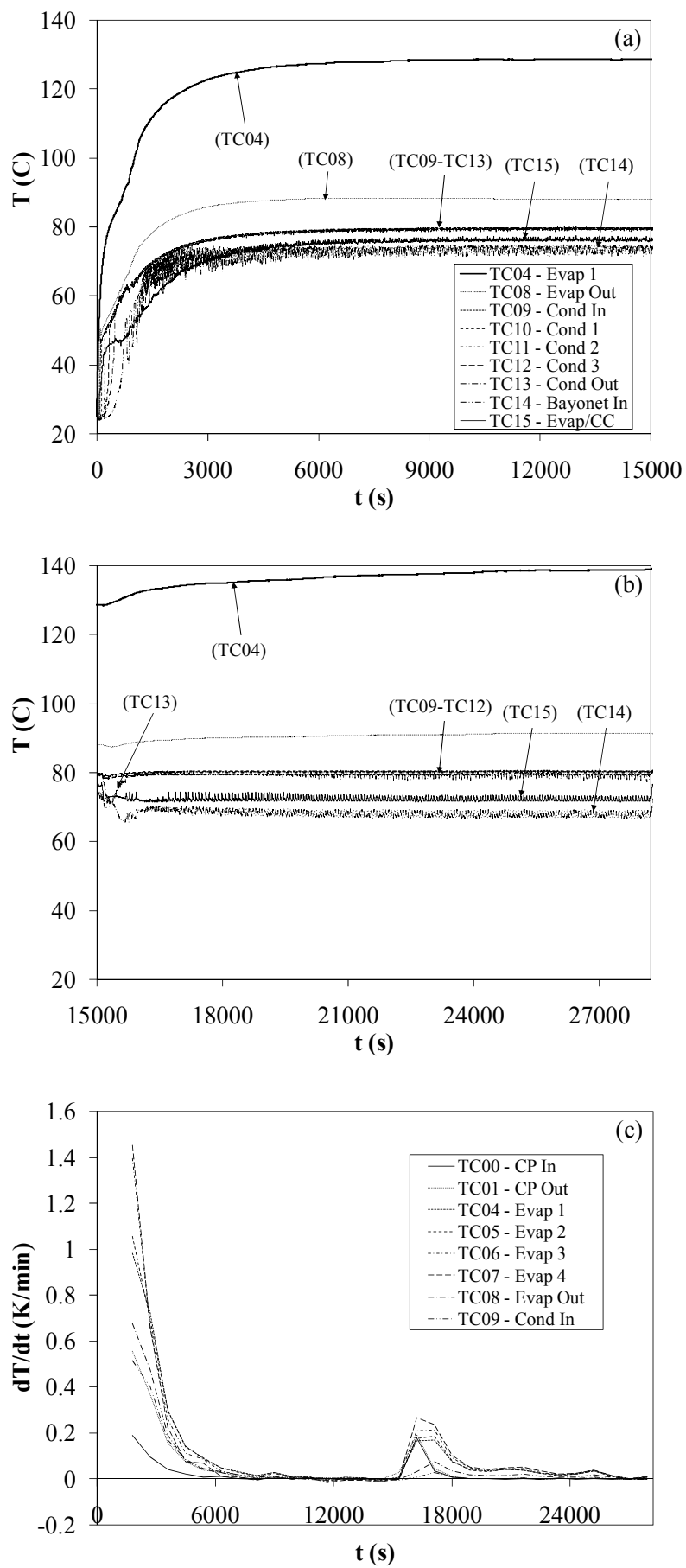

Fig. 11 Transient temperature traces of the LHP at elevated acceleration $\left(Q_{\mathrm{in}}=600 \mathrm{~W}, Q_{\mathrm{cc}}=0 \mathrm{~W}, \dot{m}_{\mathrm{cp}}=\right.$ $0.0077 \mathrm{~kg} / \mathrm{s}, 55.2 \leq \bar{T}_{\mathrm{cp}} \leq 59.7^{\circ} \mathrm{C}, 27.9 \leq T_{\mathrm{amb}} \leq 30.1^{\circ} \mathrm{C}$ ): (a) $a_{\mathrm{r}}=0.1 \mathrm{~g}$ startup phase; (b) Transition to and steady state at $a_{\mathrm{r}}=10.0 \mathrm{~g}$; (c) Transient rate of change of temperatures. 

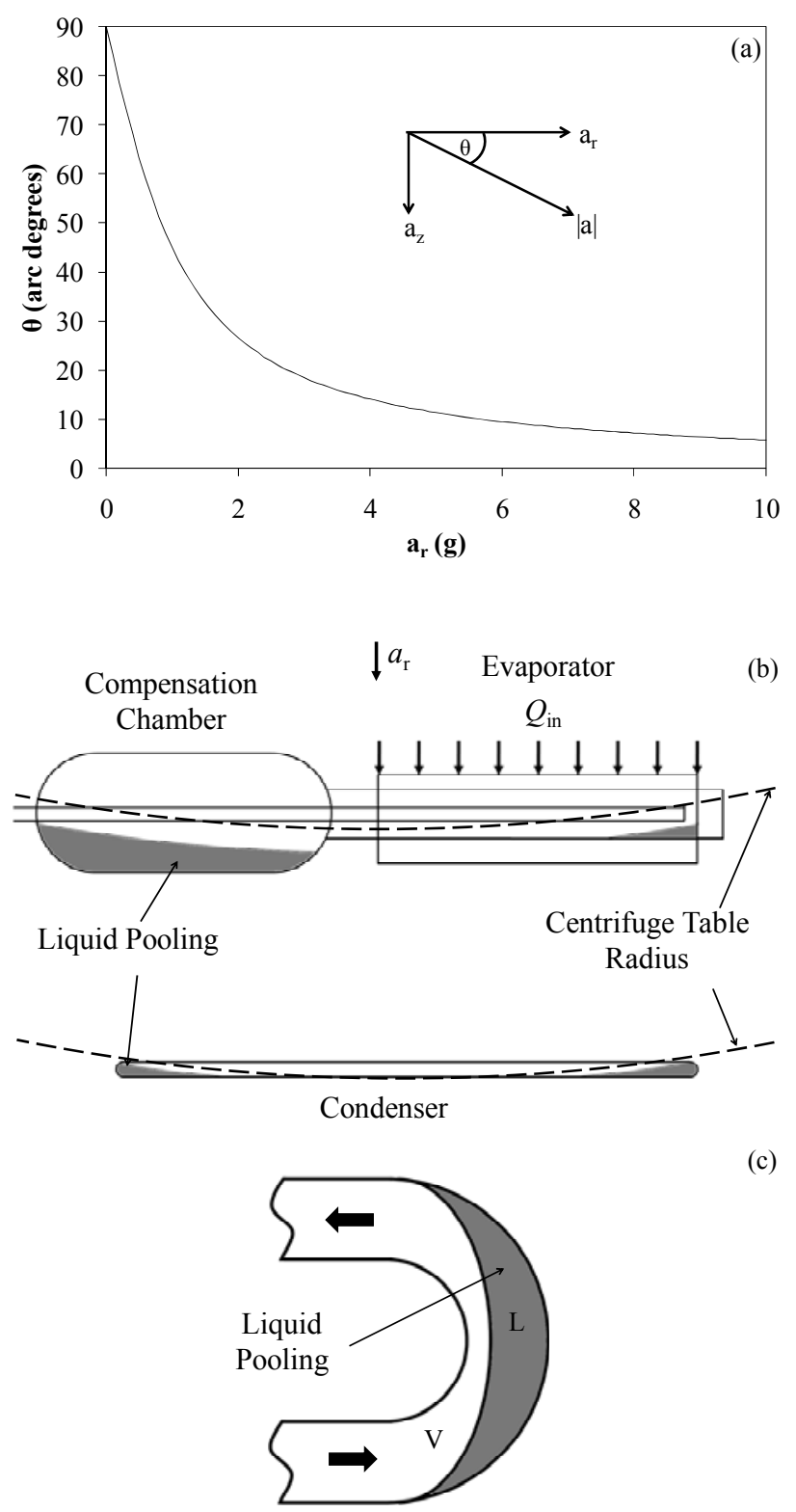

Fig. 12 Effect of resultant acceleration vector direction on fluid distribution within the LHP: (a) Resultant acceleration vector angle versus radial acceleration; (b) Liquid pooling in the evaporator, compensation chamber, and condenser under elevated acceleration (to scale, top view); (c) Liquid pooling in the condenser bends. 

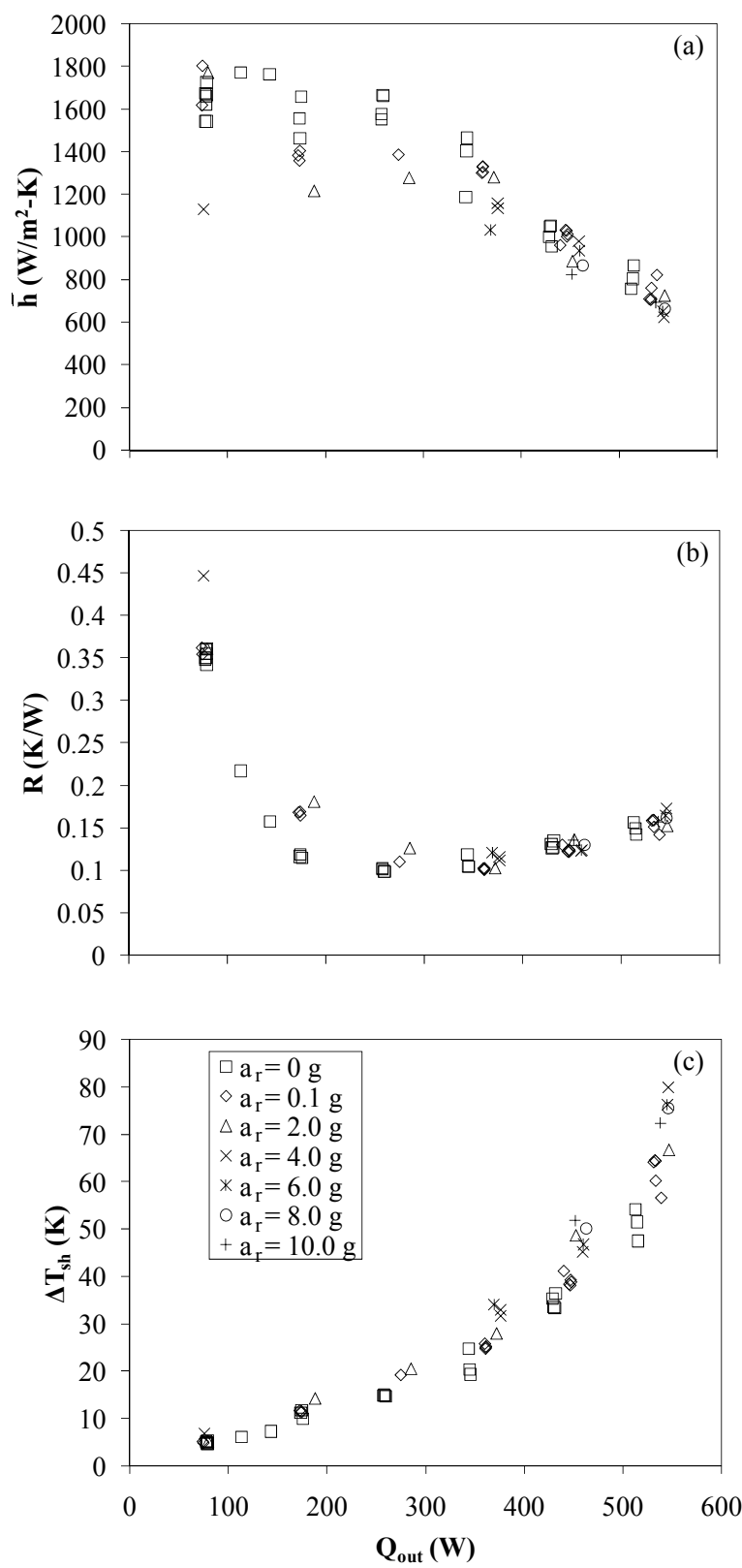

Fig. 13 Steady state performance characteristics of the LHP versus transported heat at stationary and elevated acceleration $\left(Q_{\mathrm{cc}}=0 \mathrm{~W}, \dot{m}_{\mathrm{cp}}=0.0077 \mathrm{~kg} / \mathrm{s}, 37.2 \leq \bar{T}_{\mathrm{cp}} \leq 67.7^{\circ} \mathrm{C}, 25.1 \leq T_{\mathrm{amb}} \leq 38.7^{\circ} \mathrm{C}\right)$ : (a)

Evaporative heat transfer coefficient; (b) Thermal resistance; (c) Evaporator wall superheat. 

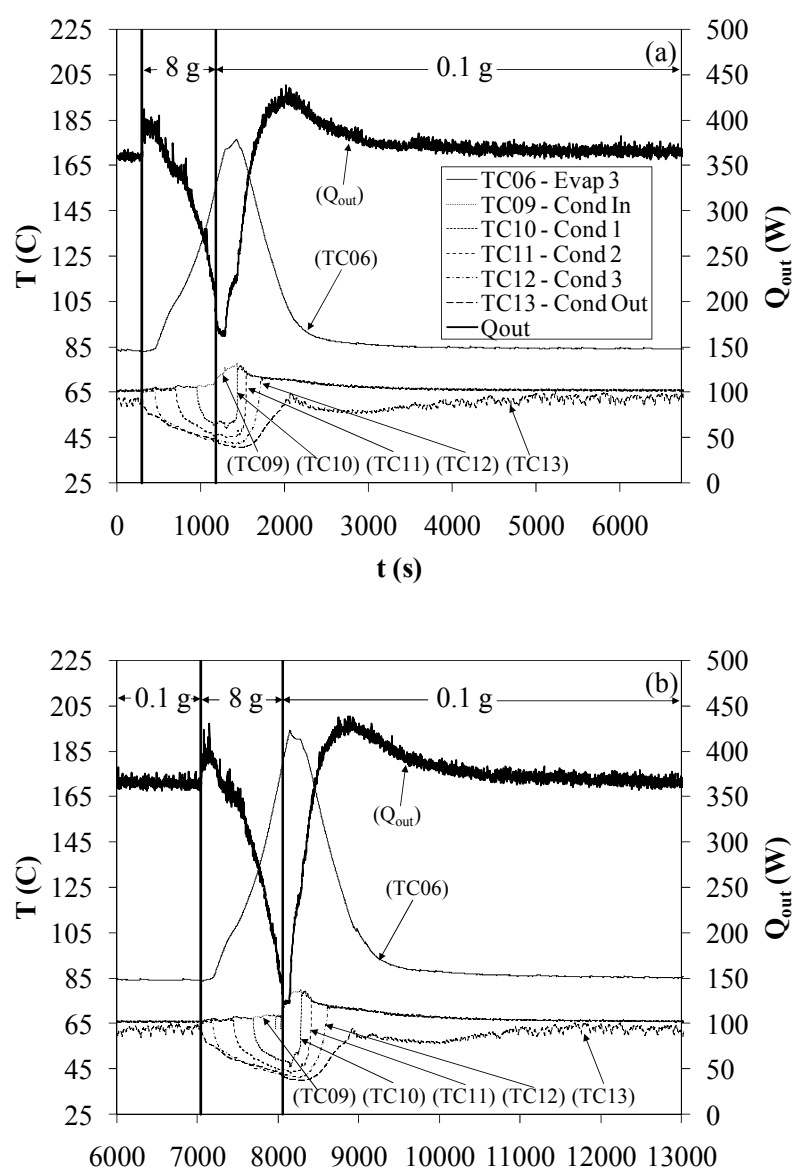

t (s)

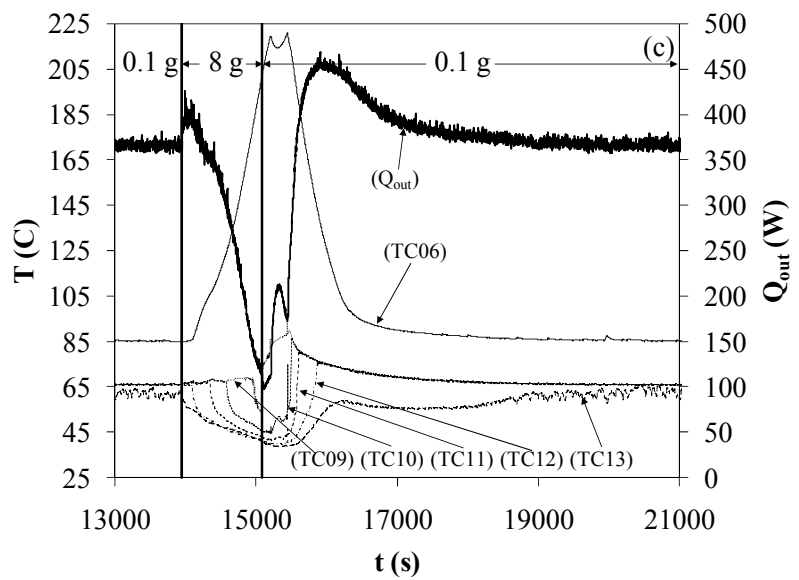

Fig. 14 Transient temperature traces of the LHP at elevated acceleration showing dry-out behavior $\left(Q_{\text {in }}=\right.$ $400 \mathrm{~W}, Q_{\mathrm{cc}}=0 \mathrm{~W}, \dot{m}_{\mathrm{cp}}=0.0077 \mathrm{~kg} / \mathrm{s}, 37.2 \leq \bar{T}_{\mathrm{cp}} \leq 59.7^{\circ} \mathrm{C}, T_{\mathrm{amb}}=28.0^{\circ} \mathrm{C}$ ): (a) $T_{\mathrm{e}, \max }=150^{\circ} \mathrm{C}$; (b) $T_{\mathrm{e}, \max }=$ $175^{\circ} \mathrm{C}$; (c) $T_{\mathrm{e}, \max }=200^{\circ} \mathrm{C}$. 

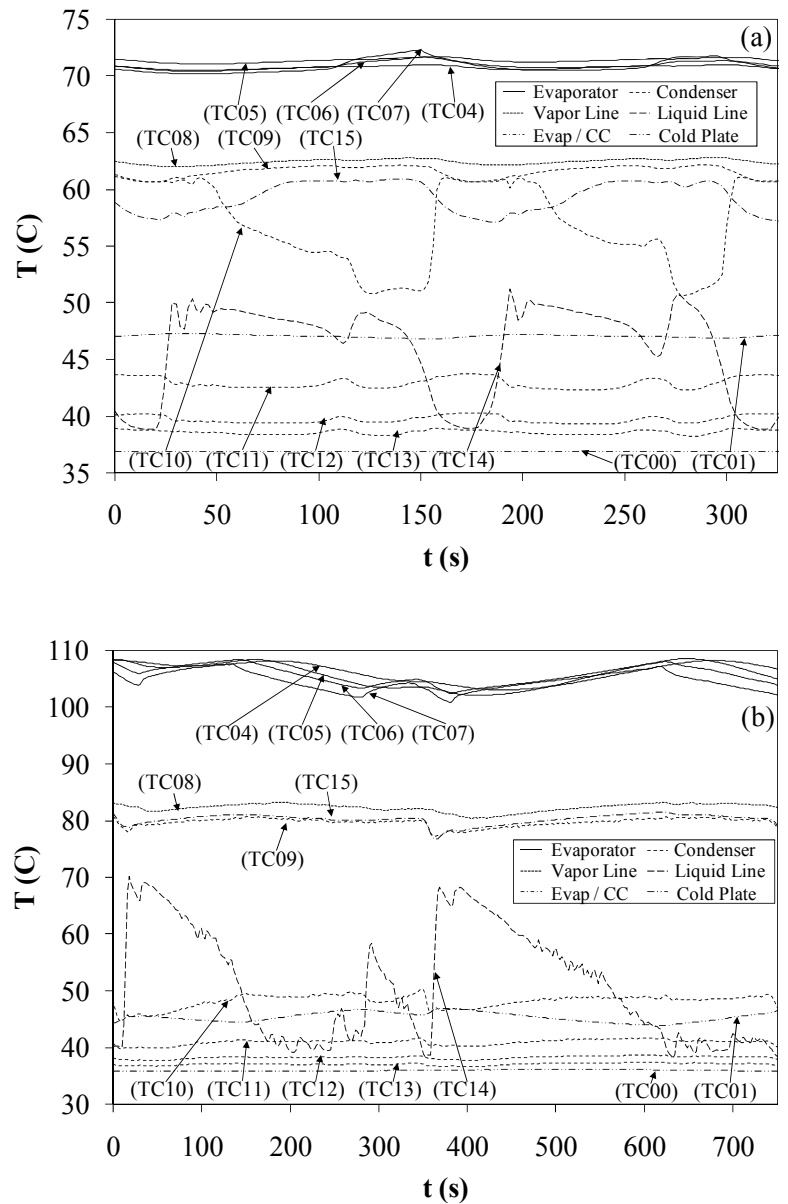

Fig. 15 Quasi-steady state temperature traces of the LHP and cold plate $\left(Q_{\mathrm{in}}=200 \mathrm{~W}, Q_{\mathrm{cc}}=0 \mathrm{~W}, \dot{m}_{\mathrm{cp}}=\right.$ $0.0077 \mathrm{~kg} / \mathrm{s}, \bar{T}_{\mathrm{cp}}=41.9^{\circ} \mathrm{C}, T_{\mathrm{amb}}=26.4^{\circ} \mathrm{C}$ ): (a) $a_{\mathrm{r}}=0.1 \mathrm{~g}$; (b) $a_{\mathrm{r}}=4.0 \mathrm{~g}$. 


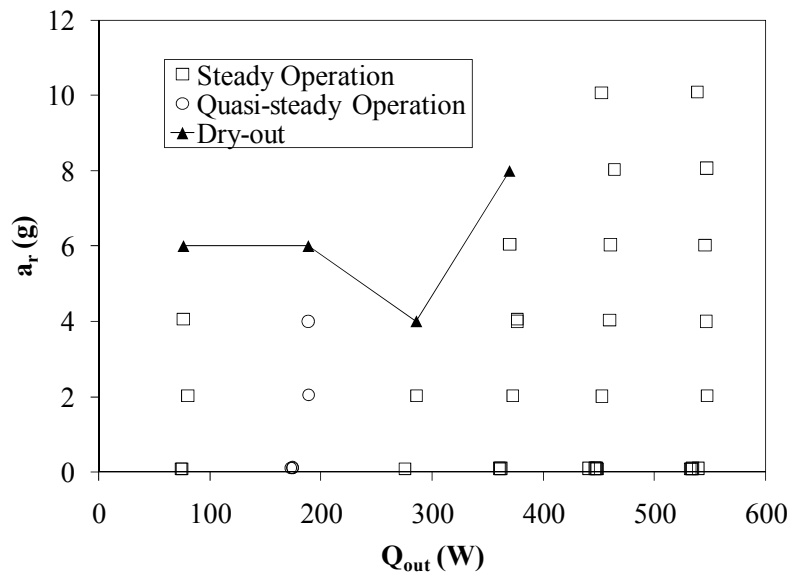

Fig. 16 Steady state performance map of the LHP relating radial acceleration and heat transported $\left(Q_{\mathrm{cc}}=\right.$ $\left.0 \mathrm{~W}, \dot{\boldsymbol{m}}_{\mathrm{cp}}=0.0077 \mathrm{~kg} / \mathrm{s}, 37.2 \leq \bar{T}_{\mathrm{cp}} \leq 59.7^{\circ} \mathrm{C}, 25.1 \leq T_{\mathrm{amb}} \leq 30.2^{\circ} \mathrm{C}\right)$. 
Table 1 LHP design summary.

\begin{tabular}{|c|c|}
\hline Requirement & Parameter \\
\hline \multicolumn{2}{|c|}{ Thermal } \\
\hline Minimum Heat Load & $500 \mathrm{~W}$ \\
\hline Minimum Heat Flux & $3 \mathrm{~W} / \mathrm{cm}^{2}$ \\
\hline Maximum Operating Temperature & $200^{\circ} \mathrm{C}$ \\
\hline Condenser Heat Sink Temperature & 5 to $140^{\circ} \mathrm{C}$ \\
\hline Tilt in One G & \pm 0 inches, horizontal \\
\hline Conductance & $50^{\circ} \mathrm{C} / \mathrm{W}$ \\
\hline Proof of Pressure Test & $3102 \mathrm{psi}\left(200^{\circ} \mathrm{C}\right)$ \\
\hline \multicolumn{2}{|c|}{ Materials } \\
\hline Evaporator Envelope Material & Titanium, CP Grade 2 \\
\hline Primary Wick Material & Titanium, CP Grade 2 \\
\hline Secondary Wick Material & Titanium, CP Grade 2 \\
\hline Transport Line Material & Titanium, CP Grade 2 \\
\hline Working Fluid & Water \\
\hline Fluid Charge & $300 \mathrm{~mL}$ \\
\hline \multicolumn{2}{|c|}{ LHP Dimensions } \\
\hline Evaporator Footprint & $20.32 \times 10.16 \mathrm{~cm}^{2}$ \\
\hline Condenser Footprint & $30.48 \times 28.58 \mathrm{~cm}^{2}$ \\
\hline Vapor Line Length & Approx. $243.8 \mathrm{~cm}$ \\
\hline Vapor Line Diameter & $0.9525 \mathrm{OD} \times 0.0889 \mathrm{~cm}$ wall \\
\hline Liquid Line Length & Approx. $335.3 \mathrm{~cm}$ \\
\hline Liquid Line Diameter & $0.6350 \mathrm{OD} \times 0.0889 \mathrm{~cm}$ wall \\
\hline Condenser Line Length & Approx. $279.4 \mathrm{~cm}$ \\
\hline Condenser Line Diameter & $0.9525 \mathrm{OD} \times 0.0889 \mathrm{~cm}$ wall \\
\hline \multicolumn{2}{|c|}{ Compensation Chamber } \\
\hline Diameter & $6.033 \mathrm{~cm} \mathrm{OD}$ \\
\hline Length & $11.43 \mathrm{~cm}$ \\
\hline Chamber Location & Coaxial with evaporator \\
\hline \multicolumn{2}{|c|}{ Primary Wick Properties } \\
\hline Effective Pore Radius & $9.1 \mu \mathrm{m}$ \\
\hline Permeability & $1.2 \times 10^{-12} \mathrm{~m}^{2}$ \\
\hline Outside Diameter & $2.286 \mathrm{~cm}$ \\
\hline Length & $20.32 \mathrm{~cm}$ \\
\hline Inside Diameter & $0.8001 \mathrm{~cm}$ \\
\hline Number of Grooves & 6 \\
\hline Groove Depth & $0.1524 \mathrm{~cm}$ \\
\hline Groove Width & $0.1524 \mathrm{~cm}$ \\
\hline \multicolumn{2}{|c|}{ Secondary Wick Properties } \\
\hline Mesh & $150 \times 150$ \\
\hline
\end{tabular}


Table 2 Summary of thermocouple locations.

\begin{tabular}{cc}
\hline Thermocouple & Location \\
\hline TC00 & Cold Plate Inlet \\
TC01 & Cold Plate Outlet \\
TC02 & PAO Flow Meter Inlet \\
TC03 & Ambient \\
TC04 & Evaporator 1 \\
TC05 & Evaporator 2 \\
TC06 & Evaporator 3 \\
TC07 & Evaporator 4 \\
TC08 & Evaporator Outlet \\
TC09 & Condenser Inlet \\
TC10 & Condenser 1 \\
TC11 12 & Condenser 2 \\
TC12 & Condenser 3 \\
TC13 & Condenser Outlet \\
TC14 & Bayonet Inlet \\
TC15 & Evaporator/CC Junction \\
\hline \hline
\end{tabular}


Table 3 Summary of Uncertainty Analysis.

\begin{tabular}{|c|c|c|}
\hline & Quantity & Uncertainty \\
\hline \multirow[t]{20}{*}{ Measured } & TC00 & $\pm 0.12^{\circ} \mathrm{C}$ \\
\hline & TC01 & $\pm 0.11^{\circ} \mathrm{C}$ \\
\hline & TC02 & $\pm 0.11^{\circ} \mathrm{C}$ \\
\hline & TC03 & $\pm 0.11^{\circ} \mathrm{C}$ \\
\hline & TC04 & $\pm 0.31^{\circ} \mathrm{C}$ \\
\hline & TC05 & $\pm 0.29^{\circ} \mathrm{C}$ \\
\hline & TC06 & $\pm 0.29^{\circ} \mathrm{C}$ \\
\hline & TC07 & $\pm 0.33^{\circ} \mathrm{C}$ \\
\hline & TC08 & $\pm 0.33^{\circ} \mathrm{C}$ \\
\hline & ТC09 & $\pm 0.29^{\circ} \mathrm{C}$ \\
\hline & TC10 & $\pm 0.29^{\circ} \mathrm{C}$ \\
\hline & TC11 & $\pm 0.29^{\circ} \mathrm{C}$ \\
\hline & $\mathrm{TC} 12$ & $\pm 0.27^{\circ} \mathrm{C}$ \\
\hline & TC13 & $\pm 0.30^{\circ} \mathrm{C}$ \\
\hline & TC14 & $\pm 0.30^{\circ} \mathrm{C}$ \\
\hline & TC15 & $\pm 0.30^{\circ} \mathrm{C}$ \\
\hline & Acceleration & $+\left(0.01+\left(a_{\mathrm{r}, \mathrm{oe}}-a_{\mathrm{r}, \mathrm{cl}}\right)\right) \mathrm{g}$ \\
\hline & & $-\left(0.01+\left(a_{\mathrm{r}, \mathrm{cl}}-a_{\mathrm{r}, \mathrm{ie}}\right)\right) \mathrm{g}$ \\
\hline & Mass flow rate & $\pm 4 \%$ \\
\hline & Voltages & $\pm(0.00025 \mathrm{~V}+0.005) \mathrm{V}$ \\
\hline \multirow[t]{2}{*}{ Constant } & Resistors & $\pm 0.0002 R \Omega$ \\
\hline & Wick $D, L$ & $\pm 0.0000254 \mathrm{~m}$ \\
\hline \multirow[t]{7}{*}{ Calculated } & $\bar{T}_{\mathrm{cp}}$ & $\pm \max \left(\Delta T_{\text {out }}, \Delta T_{\text {in }}\right)$ \\
\hline & $\bar{T}_{\mathrm{e}}$ & $\pm \max (\Delta(\mathrm{TC} 04), \Delta(\mathrm{TC} 05), \Delta(\mathrm{TC} 06), \Delta(\mathrm{TC} 07))$ \\
\hline & $\Delta T_{\mathrm{sh}}$ & $\pm 0.63^{\circ} \mathrm{C}$ \\
\hline & $C_{\mathrm{p}, \mathrm{PAO}}$ & $\pm 0.005 * C_{\mathrm{p}}\left(\bar{T}_{\mathrm{cp}}\right)$ \\
\hline & $Q_{\text {out }}$ & Eqn. (4) \\
\hline & $\bar{h}$ & Eqn. (5) \\
\hline & $R$ & Eqn. (6) \\
\hline
\end{tabular}


Table 4 The effect of compensation chamber temperature control on LHP operation $\left(Q_{\text {in }}=500 \mathrm{~W}, a_{\mathrm{r}}=0\right.$ $\left.\mathrm{g}, \dot{\boldsymbol{m}}_{\mathrm{cp}}=0.0077 \mathrm{~kg} / \mathrm{s}, \mathrm{T}_{\mathrm{amb}}=26.4^{\circ} \mathrm{C}\right)$.

\begin{tabular}{|c|c|c|c|c|c|c|c|c|}
\hline $\begin{array}{c}\text { Compensation } \\
\text { Chamber } \\
\text { Conditions }\end{array}$ & $\begin{array}{l}Q_{\mathrm{cc}} \\
(\mathrm{W})\end{array}$ & $\begin{array}{c}\overline{T_{\mathrm{e}}} \\
\left({ }^{\circ} \mathrm{C}\right)\end{array}$ & $\begin{array}{c}\bar{T}_{\mathrm{cp}} \\
\left({ }^{\circ} \mathrm{C}\right)\end{array}$ & $\begin{array}{l}T_{\mathrm{cc}} \\
\left({ }^{\circ} \mathrm{C}\right)\end{array}$ & $\begin{array}{l}Q_{\text {out }} \\
\text { (W) }\end{array}$ & $\begin{array}{c}\bar{h} \\
\left(\mathrm{~W} / \mathrm{m}^{2}-\mathrm{K}\right)\end{array}$ & $\begin{array}{c}R \\
(\mathrm{~K} / \mathrm{W})\end{array}$ & $\begin{array}{l}\text { Time to } \\
\text { Steady } \\
\text { State }\end{array}$ \\
\hline $\begin{array}{l}\text { Uninsulated, no } \\
\text { temperature control }\end{array}$ & 0 & 115 & 52.4 & 59.3 & $442 \pm 19$ & $878 \pm 37$ & $0.142 \pm 0.0060$ & $300 \mathrm{~min}$. \\
\hline $\begin{array}{l}\text { Insulated, no } \\
\text { temperature control }\end{array}$ & 0 & 107 & 52.6 & 62.9 & $447 \pm 19$ & $1050 \pm 51$ & $0.122 \pm 0.0052$ & 375 min. \\
\hline $\begin{array}{l}\text { Insulated, } \\
\text { temperature } \\
\text { controlled to } T_{\mathrm{cc}}= \\
72.8^{\circ} \mathrm{C} \text {, } \\
\text { simultaneous heat } \\
\text { input startup }\end{array}$ & 20 & 103 & 53.5 & 72.8 & $470 \pm 20$ & $1340 \pm 77$ & $0.106 \pm 0.0045$ & $310 \mathrm{~min}$. \\
\hline $\begin{array}{l}\text { Insulated, } \\
\text { temperature } \\
\text { controlled to } T_{\mathrm{cc}}= \\
72.8^{\circ} \mathrm{C} \text {, } \\
\text { preconditioned } \mathrm{CC}\end{array}$ & $\begin{array}{l}100 \\
\text { to } \\
20\end{array}$ & 103 & 53.4 & 72.8 & $467 \pm 19$ & $1350 \pm 77$ & $0.106 \pm 0.0045$ & $250 \mathrm{~min}$. \\
\hline
\end{tabular}




\section{References}

[1] Maidanik, J., "Review: Loop Heat Pipes," Applied Thermal Engineering, Vol. 25, 2005, pp. 635-657.

[2] Maidanik, J., Vershinin, S., Kholodov, V., Dolgirev, J., "Heat Transfer Apparatus," United States Patent 4,515,209, 1985.

[3] Hoang, T., Ku, J., "Transient Modeling of Loop Heat Pipes," AIAA Paper 2003-6082, August 2003

[4] Ku, J., Ottenstein, L., Kaya, T., Rogers, P., Hoff, C., "Testing of a Loop Heat Pipe Subjected to Variable Accelerating Forces, Part 1: Start-Up," SAE Paper 2000-01-2488, July 2000.

[5] Ku, J., Ottenstein, L., Kaya, T., Rogers, P., Hoff, C., "Testing of a Loop Heat Pipe Subjected to Variable Accelerating Forces, Part 2: Temperature Stability," SAE Paper 2000-01-2489, July 2000

[6] Ponnappan, R., Yerkes, K., Chang, W., Beam, J., "Analysis and Testing of Heat Pipe in Accelerating Environment," Proceedings of the 8th International Heat Pipe Conference, Beijing, China, 1992, pp. B-191 - B-19-6.

[7] Yerkes, K., Beam, J., "Arterial Heat Pipe Performance in a Transient Heat Flux and Body Force Environment," SAE Paper 921944, October 1992.

[8] Thomas, S., Yerkes, K., "Quasi-Steady-State Performance of a Heat Pipe Subjected to Transient Acceleration Loadings," AIAA Journal of Thermophysics, Vol. 11, No. 2, 1996, pp. 306-309.

[9] Thomas, S., Klasing, K., Yerkes, K., "The Effects of Transverse Acceleration-Induced Body Forces on the Capillary Limit of Helically Grooved Heat Pipes," ASME Journal of Heat Transfer, Vol. 120, 1998, pp. 441-451.

[10]Zaghdoudi, M., Sarno, C., "Investigation of the Effects of Body Force Environment on Flat Heat Pipes." AIAA Journal of Thermophysics and Heat Transfer, Vol. 15, No. 4, 2001, pp. 384-394.

[11] Anderson, W., Dussinger, P., Bonner, R., and Sarraf, D., "High Temperature Titanium-Water and MonelWater Heat Pipes," AIAA Paper 2006-4113, June 2006.

[12] Anderson, W., Bonner, R., Dussinger, P., Hartenstine, J., and Sarraf, D., "Intermediate Temperature Fluids Life Tests-Experiments," AIAA Paper 2007-4808, June 2007.

[13] Fleming, A., "Aircraft Thermal Management Using Loop Heat Pipes," Master's Thesis, Department of Mechanical and Materials Engineering, Wright State University, Dayton, OH, March 2009.

[14] Ghajar, A., Tang, W., Beam, J., "Comparison of Hydraulic and Thermal Performance of PAO and Coolanol 25R," AIAA Paper 94-1965, June 1994.

[15] Douglas, D., Ku, J., Kaya, T., "Testing of the Geoscience Laser Altimeter System (GLAS) Prototype Loop Heat Pipe," AIAA Paper 99-0473, January 1999.

[16] Cimbala, J., Brenizer, J., Chuang, A., Hanna, S., Conroy, C., El-Ganayni, A., Riley, D., "Study of a Loop Heat Pipe Using Neutron Radiography," Applied Radiation and Isotopes, Vol. 61, 2004, pp. 701-705.

[17] Ku, J. "Operating Characteristics of Loop Heat Pipes." SAE Paper 1999-01-2007, July 1999.

[18] Incropera, F., DeWitt, D., Fundamentals of Heat and Mass Transfer, Wiley, New York City, 2002.

[19] Boyer, R., Welsch, G., Collings, E., Materials Properties Handbook: Titanium Alloys, ASM International, Materials Park, OH, 1994. 